\title{
Dynamics of a rolling disk in the presence of dry friction
}

\section{Journal Article}

Author(s):

Le Saux C.; Leine, R.I.; Glocker, Christoph

Publication date:

2005-02

Permanent link:

https://doi.org/10.3929/ethz-b-000033234

Rights / license:

In Copyright - Non-Commercial Use Permitted

Originally published in:

Journal of Nonlinear Science 15(1), https://doi.org/10.1007/s00332-004-0655-4 
J. Nonlinear Sci. Vol. 15: pp. 27-61 (2005)

DOI: $10.1007 / \mathrm{s} 00332-004-0655-4$

\title{
Nonlinear
} Science

(C) 2005 Springer Science+Business Media, Inc.

\section{Dynamics of a Rolling Disk in the Presence of Dry Friction}

\author{
C. Le Saux ${ }^{1}$, R. I. Leine ${ }^{1}$, and C. Glocker ${ }^{1}$ \\ 1 Institute of Mechanical Systems, Centre of Mechanics, ETH Zentrum, CH-8092 Zurich, \\ Switzerland \\ E-mail: remco.leine@imes.mavt.ethz.ch; christoph.glocker@imes.mavt.ethz.ch
}

Received July 20, 2004; accepted November 29, 2004

Online publication March 11, 2005

Communicated by G. Stépán

Summary. In this paper we are interested in the dynamics and numerical treatment of a rolling disk on a flat support. The objective of the paper is to develop a numerical model which is able to simulate the dynamics of a rolling disk taking into account various kinds a friction models (resistance against sliding, pivoting and rolling). A mechanical model of a rolling disk is presented in the framework of Non-smooth Dynamics and Convex Analysis. In an analytical study, approximations are derived for the energy decay of the system during the final stage of the motion for various kinds of frictional dissipation models. Finally, the numerical and analytical results are discussed and compared with experimental results available in literature.

AMS Subject Classification. 70E18, 70F35, 70E55, 74M10

Key words. unilateral contact, friction, time-stepping method, Euler disk, set-valued force laws

\section{Introduction}

How can moving bodies be stopped in finite time? Many answers different from classical solutions are provided by nonsmooth dynamics, which deals with measure differential inclusions from time-evolution problems in mechanics. The most familiar examples of such systems are collisions of rigid bodies and Coulomb's law of dry friction. Nonsmooth dynamics, however, is much more than friction and impacts. It provides an extended concept of classical analytical mechanics with all its subdisciplines and modern extensions, such as multibody dynamics and robotics, by allowing for set-valued constitutive laws 
and discontinuities in the state variables as functions of time. Set-valued constitutive laws provide a sound basis for switching on and off various kinds of constraints in a most consistent way, where the switching rules are embedded in the constitutive laws themselves. One important subclass of such constitutive laws are those expressed by normal cone inclusions from convex analysis, as used in this paper, because they generalize the dynamics on manifolds to manifolds with boundary. As one of the most demanding examples, the classical problem of a rolling disk is chosen with the main emphasis being the numerical study of the dissipation mechanisms generated by different kinds of set-valued constitutive laws at the contact point.

The theoretical framework developed during the last decades in the fields of numerical analysis and nonsmooth dynamics provides the basis for the efficient numerical simulation of multibody systems submitted to multiple unilateral contact constraints with friction. When dealing with multibody systems submitted to many unilateral contact constraints, the so-called time-stepping approach has proven its efficiency and robustness [10], [19]. The time-stepping method permits us to study various kinds of mechanical systems in civil engineering (granular materials), dynamics of machines (turbine blade dampers), robotics (walking robots), and mechanisms (electrical circuit-breakers). However, unilateral contact between an object with a flat side of rounded contour and a plane, like that of a bottle on a table, is still a topic of research. Such a type of contact can for instance be found in grinding machines and the transportation of cylindrical objects on a conveyor belt. Systems with such a type of contact undergo a specific type of motion, as is described by a scientific toy called the "Euler Disk." The Euler disk consists of a metal disk, about $75 \mathrm{~mm}$ in diameter and $12 \mathrm{~mm}$ thick, which can spin on a slightly concave mirror, called the support. More commonly, a similar kind of motion, although with more damping, is that of a coin spinning on a table. This type of motion involves an energy decrease to zero in a finite time accompanied by a certain kind of singularity. In the course of the motion, both the inclination of the disk with respect to the support and its angular velocity decrease to zero, while the relative velocity of the contact point with respect to the disk tends to infinity in the final stage of the motion. The spinning disk on a flat support, which constitutes the simplest example of this specific type of motion regarding the shape of the body, has often been discussed theoretically since the nineteenth century up to now [3], [4], [7], [11], [15], [16], [21], [24].

O'Reilly [21] studies the dynamics of the "rolling disk," a disk which is purely rolling without dissipation, and of the "sliding disk," a disk without friction. Following Appell [3], Chaplygin [6], and Korteweg [12], O'Reilly is able to find closed-form solutions using Legendre functions of complex degree. Bifurcation diagrams are presented in [21], but the bifurcation parameters (A and B) are very hard to interpret physically.

Kessler and O'Reilly [11] discuss the dynamics of the Euler disk under the influence of dissipation. A friction model is taken into account which models sliding friction as well as a form of rolling friction and drilling friction (but the different kinds of dissipation are uncoupled). The sliding friction model has a static and a dynamic friction coefficient, and the numerical results therefore show stick-slip-like behaviour. The numerical simulations show an asymptotic energy decrease, i.e., the disk does not stop in finite time. 
In this paper we are interested in the dynamics and numerical treatment of a rolling disk on a flat support. The objective of the paper is twofold:

1. To develop a numerical model which is able to simulate the dynamics of a rolling disk taking into account

(a) unilateral contact with a flat rigid support,

(b) collisions, stick-slip transitions, and other frictional effects between disk and support,

(c) motion without contact, motion with rim-support contact, motion with facesupport contact (i.e., "motion in the plane") and transitions from one to the other,

(d) a nonsingular parameterization of the disk;

2. To study the dynamics of a rolling disk

(a) by the numerical model developed in 1 for various frictional dissipation mechanisms (resistance against sliding, pivoting, and rolling),

(b) by an analytical study,

(c) considering experimental results available in literature.

In Section 2, we present a mechanical model of a rolling disk in the framework of nonsmooth dynamics and convex analysis [5], [8], [14], [18]. We use Euler parameters (quaternions) for the description of the orientation of the disk. We model the disk with three unilateral contact constraints of the type point-surface in order to simulate a static equilibrium of the disk lying horizontally on the support as well as motion in the plane. The constitutive laws associated with the frictional unilateral contact constraints are set-valued force laws that account for resistance against sliding, pivoting, and rolling.

A numerical algorithm of the time-stepping type is briefly presented in Section 3. The numerical results, obtained with various combinations of frictional dissipation models, are presented in Section 4. An analytical study of the rolling motion of a disk is given in Section 5. Approximations are derived for the energy decay of the system during the final stage of the motion for various kinds of frictional dissipation models. Finally, the numerical and analytical results are discussed and compared with experimental results available in literature (Section 6).

\section{Mechanical Model}

For displaying various terms in the three-dimensional Euclidean space, we use the following notation. Different points are denoted by uppercase roman symbols, such as $A$ or $B$. The displacement of point $B$ relative to a frame in point $A$ is expressed by the vector $\boldsymbol{r}_{A B}$. The term $\boldsymbol{v}_{B}$ denotes the velocity of point $B$, i.e., $\boldsymbol{v}_{B}=\dot{\boldsymbol{r}}_{A B}$ if $A$ is not moving. Only orthonormal frames are used, which we write as $C=\left(B, \boldsymbol{e}_{x}^{C}, \boldsymbol{e}_{y}^{C}, \boldsymbol{e}_{z}^{C}\right)$. The angular velocity of frame $C$ relative to another frame $D$ is denoted by $\omega_{D C}$. The angular velocity of a body is thus $\omega_{I B}$, if $B$ denotes a body-fixed frame and $I$ is an inertial frame. The components of a vector $\boldsymbol{a}$ associated with a frame $D$ are denoted ${ }_{D} \boldsymbol{a}$. For example, ${ }_{I} \boldsymbol{\omega}_{D C}$ would then be the 3-tuple of the angular velocity of frame $C$ relative to frame $D$, expressed in the inertial frame $I$. Differentiation with respect to time is indicated by a dot. For example, ${ }_{B} \dot{\boldsymbol{r}}_{A D}$ means $\frac{d}{d t}\left({ }_{B} \boldsymbol{r}_{A D}\right)$, i.e., the three entries of ${ }_{B} \boldsymbol{r}_{A D}$ are differentiated with respect to time. The velocity ${ }_{B} \boldsymbol{v}_{D}$ of point $D$ written down in frame $B$ is then obtained 


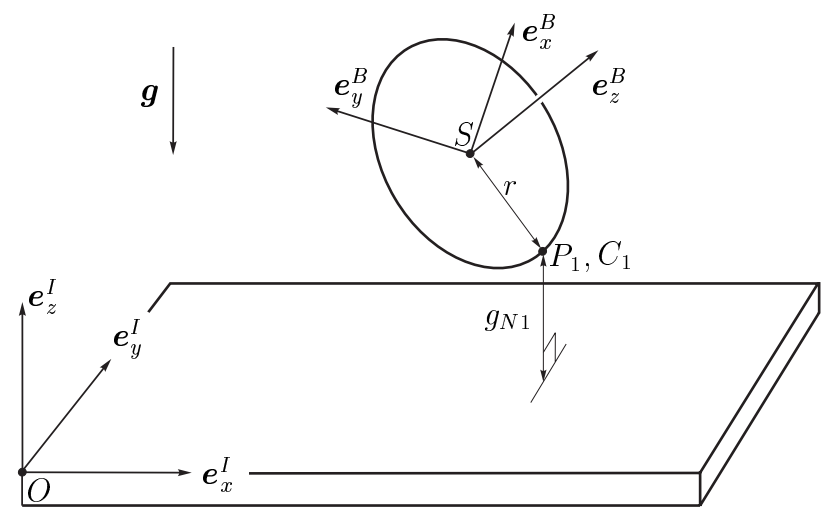

Fig. 1. Mechanical system.

by Euler's formula ${ }_{B} \boldsymbol{v}_{D}={ }_{B} \dot{\boldsymbol{r}}_{A D}+{ }_{B} \boldsymbol{\omega}_{I B} \times{ }_{B} \boldsymbol{r}_{A D}$, if $A$ is not moving and $I$ denotes the inertial frame. The general version of Euler's formula reads ${ }_{B}(\dot{a})={ }_{B} \dot{a}+{ }_{B} \boldsymbol{\omega}_{I B} \times{ }_{B} \boldsymbol{a}$ and is applied in Section 5 on the linear momentum $\overline{\boldsymbol{p}}$ and on the angular momentum $\overline{\boldsymbol{N}}_{S}$.

The mechanical system under consideration consists of a disk $\Omega$ with radius $r$, mass $m$, and centre of mass $S$, which can be in contact with a table during its motion. The system is modelled as simply as possible, in order to show only the main physical phenomena of interest, and the disk is therefore considered to be infinitely thin. Both the disk and table are considered to be perfectly rigid. An absolute coordinate frame $I=\left(O, \boldsymbol{e}_{x}^{I}, \boldsymbol{e}_{y}^{I}, \boldsymbol{e}_{z}^{I}\right)$ is attached to the table, and a body fixed coordinate frame $B=\left(S, \boldsymbol{e}_{x}^{B}, \boldsymbol{e}_{y}^{B}, \boldsymbol{e}_{z}^{B}\right)$ is attached to the disk such that $\boldsymbol{e}_{z}^{B}$ is the axis of revolution (see Figure 1). The disk has principal moments of inertia $A=B=\frac{m r^{2}}{4}$ and $C=\frac{m r^{2}}{2}$ with respect to $S$ along the axes $\left(\boldsymbol{e}_{x}^{B}, \boldsymbol{e}_{y}^{B}, \boldsymbol{e}_{z}^{B}\right)$ respectively. The inertia tensor of the disk with respect to the centre of mass $S$ is denoted by $\Theta_{S}$. Gravity is denoted by $\boldsymbol{g}$.

\subsection{System Parametrization}

The disk is allowed to undergo arbitrary rotations. Every description of the orientation of a rigid body based on three parameters includes a singularity for some orientation. We therefore parameterize the orientation of the disk with Euler parameters (unit quaternions).

For each possible configuration of the body, the absolute orientation of $\Omega$ may be defined by the coordinate transformation $\mathcal{R}:\left(\boldsymbol{e}_{x}^{I}, \boldsymbol{e}_{y}^{I}, \boldsymbol{e}_{z}^{I}\right) \mapsto\left(\boldsymbol{e}_{x}^{B}, \boldsymbol{e}_{y}^{B}, \boldsymbol{e}_{z}^{B}\right)$, which can be accomplished by a finite rotation of an angle $\chi$ around an axis that is specified by the unit vector $\boldsymbol{n}$. We denote by $n_{i}$ the three components of ${ }_{I} \boldsymbol{n}$, i.e., the three components of $\boldsymbol{n}$ in the basis $\left(\boldsymbol{e}_{x}^{I}, \boldsymbol{e}_{y}^{I}, \boldsymbol{e}_{z}^{I}\right)$. Each rotation $(\boldsymbol{n}, \chi)$ is associated with a unit quaternion, represented in the following by the $4 \times 1$ tuple $\boldsymbol{p}$ of which the components, $e_{0}=\cos \frac{\chi}{2}$ and $e_{i}=n_{i} \sin \frac{\chi}{2}$ for $i=1,2,3$, are called the Euler parameters [22]. We will use the abbreviation $\boldsymbol{e}=\left[e_{1}, e_{2}, e_{3}\right]^{\mathrm{T}}$. The Euler parameters fulfill the relationship

$$
\boldsymbol{p}^{\mathrm{T}} \boldsymbol{p}=1, \quad \text { with } \boldsymbol{p}=\left[\begin{array}{ll}
e_{0} & \boldsymbol{e}^{\mathrm{T}}
\end{array}\right]^{\mathrm{T}} .
$$


The rotation transformation $\mathcal{R}$, which identifies with a linear mapping with associated matrix $\boldsymbol{R}=\left[R_{i j}\right]=\left(2 e_{0}^{2}-1\right) \boldsymbol{I}_{3}+2\left(\boldsymbol{e} \boldsymbol{e}^{\mathrm{T}}+e_{0} \tilde{\boldsymbol{e}}\right)$, relates the global components ${ }_{I} \boldsymbol{s}$ of a vector $\boldsymbol{s}$ to its local components ${ }_{B} \boldsymbol{S}$ as ${ }_{I} \boldsymbol{S}=\boldsymbol{R}_{B} \boldsymbol{s}$. We introduce the following two $3 \times 4$ matrices $\boldsymbol{H}$ and $\overline{\boldsymbol{H}}$ such that

$$
\boldsymbol{H}=\left[\begin{array}{ll}
-\boldsymbol{e} & \tilde{\boldsymbol{e}}+e_{0} \boldsymbol{I}
\end{array}\right], \quad \overline{\boldsymbol{H}}=\left[\begin{array}{ll}
-\boldsymbol{e} & -\tilde{\boldsymbol{e}}+e_{0} \boldsymbol{I}
\end{array}\right], \quad \boldsymbol{R}=\boldsymbol{H} \overline{\boldsymbol{H}}^{\mathrm{T}},
$$

with $\tilde{\boldsymbol{a}} \boldsymbol{b}=\boldsymbol{a} \times \boldsymbol{b}$, in which $\tilde{\boldsymbol{a}}$ is a skew-symmetric matrix. We parameterize the body $\Omega$ using a set of seven generalized coordinates composed of three translational coordinates, which are the absolute coordinates of the centre of mass $S$ (represented by ${ }_{I} \boldsymbol{r}_{O S}$ ), and four rotational coordinates, which are the Euler parameters (represented by $\boldsymbol{p}$ ). Introducing the generalized coordinate vector

$$
\boldsymbol{q}=\left[\begin{array}{c}
{ }_{I} \boldsymbol{r}_{O S} \\
\boldsymbol{p}
\end{array}\right] \in \mathbb{R}^{7},
$$

a Lagrangian description of the motion can be written in the form

$$
\forall M \in \Omega, \quad{ }_{I} \boldsymbol{r}_{O M}(\boldsymbol{q})={ }_{I} \boldsymbol{r}_{O S}+\boldsymbol{R}_{B} \boldsymbol{r}_{S M} .
$$

Equation (2.4) must be considered together with the constraint (2.1). The differentiation of the relation (2.4) leads to the Eulerian description of the motion

$$
\forall M \in \Omega, \quad{ }_{I} \dot{\boldsymbol{r}}_{O M}={ }_{I} \dot{\boldsymbol{r}}_{O S}+\dot{\boldsymbol{R}}_{B} \boldsymbol{r}_{M S},
$$

with the relations

$$
\dot{\boldsymbol{R}}={ }_{I} \tilde{\boldsymbol{\omega}}_{I B} \boldsymbol{R}, \quad \dot{\boldsymbol{R}}=\boldsymbol{R}_{B} \tilde{\boldsymbol{\omega}}_{I B},
$$

where $\tilde{\boldsymbol{\omega}}_{I B}$ is the skew-symmetric operator associated with the absolute angular velocity of the body $\boldsymbol{\omega}_{I B}$. Subsequently, we introduce the generalized velocity vector $\boldsymbol{u}$, which gathers the absolute coordinates of the global velocity of the centre of mass ${ }_{I} \dot{\boldsymbol{r}}_{O S}$ and the local coordinates of the absolute angular velocity vector ${ }_{B} \boldsymbol{\omega}_{I B}$,

$$
\boldsymbol{u}=\left[\begin{array}{c}
{ }_{I} \dot{\boldsymbol{r}}_{O S} \\
{ }_{B} \boldsymbol{\omega}_{I B}
\end{array}\right] \in \mathbb{R}^{6} .
$$

In the following, we will use the following relationships between the angular velocity vector and the derivative of the Euler parameters [22]:

$$
\begin{aligned}
{ }_{I} \boldsymbol{\omega}_{I B}=2 \boldsymbol{H} \dot{\boldsymbol{p}}, & 2 \dot{\boldsymbol{p}}=\boldsymbol{H}^{\mathrm{T}}{ }_{I} \boldsymbol{\omega}_{I B}, \\
{ }_{B} \boldsymbol{\omega}_{I B}=2 \overline{\boldsymbol{H}} \dot{\boldsymbol{p}}, & 2 \dot{\boldsymbol{p}}=\overline{\boldsymbol{H}}^{\mathrm{T}}{ }_{B} \boldsymbol{\omega}_{I B},
\end{aligned}
$$

where the components of $\dot{\boldsymbol{p}}$ are not independent according to relation

$$
\boldsymbol{p}^{\mathrm{T}} \dot{\boldsymbol{p}}=0
$$

deduced from the differentiation of (2.1). 


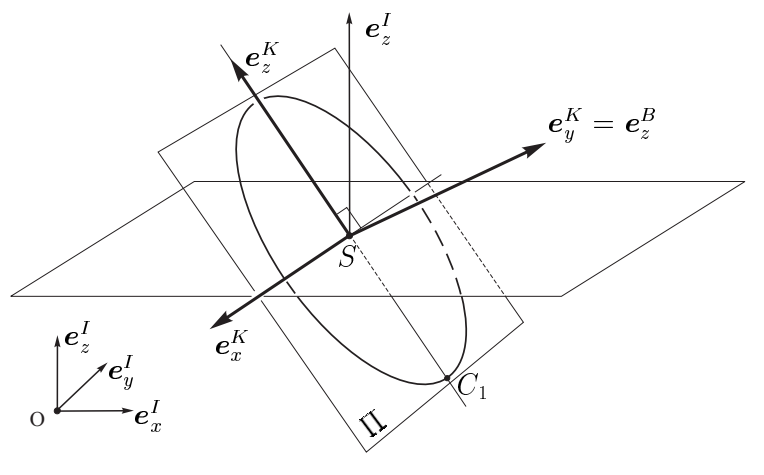

(a)

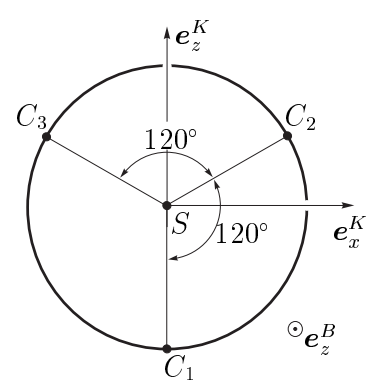

(b)

Fig. 2. Contacts point $C_{1}, C_{2}$, and $C_{3}$.

\subsection{Contact Kinematics}

During the time-evolution of the system, the disk can be in contact with the table. The unilateral contact between disk and table is assumed to be of the type point-surface. A single contact constraint is not able to describe the static equilibrium of a disk lying horizontally on the table. We therefore model the disk with three unilateral contact constraints. For an arbitrary configuration of the disk, we define three points on the contour of the disk which are candidates to contact. Subsequently, we derive the normal gap functions $g_{N j}(\boldsymbol{q})$ associated with the three unilateral contact constraints under consideration $(j=1,2,3)$. It must hold that $g_{N j}(\boldsymbol{q}) \geq 0$ to avoid penetration.

2.2.1. Derivation of Gap Functions. We first consider a nonhorizontal configuration of the disk $\left(R_{33} \neq 1\right)$. We define the point $C_{1}$ as the point on the contour of the disk which has a minimal height with respect to the plane of the table. If the disk is above the table, then $C_{1}$ is the closest point to the table. Let $\boldsymbol{e}_{x}^{K}$ be a unit directional vector of the intersection line of the plane $\Pi$ of the disk and the horizontal plane $\left(S, \boldsymbol{e}_{x}^{I}, \boldsymbol{e}_{y}^{I}\right)$ (see Figure 2a):

$$
\boldsymbol{e}_{x}^{K}=-\frac{\boldsymbol{e}_{z}^{I} \times \boldsymbol{e}_{z}^{B}}{\left\|\boldsymbol{e}_{z}^{I} \times \boldsymbol{e}_{z}^{B}\right\|} \quad \text { with } \quad\left\|\boldsymbol{e}_{z}^{I} \times \boldsymbol{e}_{z}^{B}\right\|=\sqrt{1-R_{33}^{2}} .
$$

The direction in the plane $\Pi$ with the largest inclination with respect to the plane $\left(S, \boldsymbol{e}_{x}^{I}, \boldsymbol{e}_{y}^{I}\right)$ is characterized by the unit vector $\boldsymbol{e}_{z}^{K}$

$$
\boldsymbol{e}_{z}^{K}=-\boldsymbol{e}_{z}^{B} \times \boldsymbol{e}_{x}^{K}=\frac{1}{\sqrt{1-R_{33}^{2}}}\left(\boldsymbol{e}_{z}^{I}-R_{33} \boldsymbol{e}_{z}^{B}\right)
$$

The point $C_{1}$ on the contour of the disk that has a minimal height with respect to the plane is defined by

$$
\boldsymbol{r}_{S C_{1}}=-r \boldsymbol{e}_{z}^{K}
$$


Based on the definition of $C_{1}$, we introduce two other points, $C_{2}$ and $C_{3}$, on the contour of the disk, which are candidates of contact (Figure 2b):

$$
\boldsymbol{r}_{S C_{2}}=-\frac{1}{2} \boldsymbol{r}_{S C_{1}}+\frac{\sqrt{3}}{2}\left(\boldsymbol{e}_{z}^{B} \times \boldsymbol{r}_{S C_{1}}\right), \quad \boldsymbol{r}_{S C_{3}}=-\frac{1}{2} \boldsymbol{r}_{S C_{1}}-\frac{\sqrt{3}}{2}\left(\boldsymbol{e}_{z}^{B} \times \boldsymbol{r}_{S C_{1}}\right) .
$$

The points $C_{1}, C_{2}$, and $C_{3}$ fulfill the equation $\boldsymbol{r}_{S C_{3}}=-\boldsymbol{r}_{S C_{1}}-\boldsymbol{r}_{S C_{2}}$. The gap functions $g_{N j}$, associated with the three contact points $C_{j}$, with $\boldsymbol{r}_{C_{j}}=\boldsymbol{r}_{S}+\boldsymbol{r}_{S C_{j}}$, are defined as $g_{N j}(\boldsymbol{q})=\boldsymbol{e}_{z}^{I} \cdot \boldsymbol{r}_{C_{j}}(j=1,2,3)$ and can be expressed in the form

$$
g_{N 1}(\boldsymbol{q})=z_{S}-r \sqrt{1-R_{33}^{2}}, \quad g_{N 2}(\boldsymbol{q})=g_{N 3}(\boldsymbol{q})=z_{S}+\frac{r}{2} \sqrt{1-R_{33}^{2}} .
$$

The functions $g_{N j}$ (2.15) are continuous functions in $\boldsymbol{q}$.

If the disk is parallel to the table, then the proximal point cannot be uniquely defined because all points on the disk have the same height $z_{S}$, and the configuration is said to be singular. In this case $\left(R_{33}=1, g_{N 1}=g_{N 2}=g_{N 3}=z_{S}\right)$, the functions $\boldsymbol{r}_{S C_{j}}(\boldsymbol{q})$ ( $j=1,2,3)$, given by the relations (2.13) and (2.14), are not defined. The points $C_{1}$, $C_{2}$, and $C_{3}$ can therefore, a priori, be chosen arbitrarily on the contour of the disk, while fulfilling (2.14). When, at a time-instant $t^{*}$, the disk passes a horizontal singularity position during its motion, we define the points $C_{1}, C_{2}$, and $C_{3}$ by the continuity condition $\boldsymbol{r}_{S C_{j}}^{+}\left(t^{*}\right)=\boldsymbol{r}_{S C_{j}}^{-}\left(t^{*}\right)$ for $j=1,2,3$, where the upper indices + and - denote the right and left limit at $t^{*}$ with respect to time $t$. A particular case is discussed in Section 2.2.2.

2.2.2. Derivation of Relative Velocities at the Contact Points. Consider the constrained motion of the disk, such that it is in contact with the table at a single point $C_{1}$ $\left(g_{N 1}=0, g_{N 2}=g_{N 3}>0\right)$. The point $C_{1}$, defined by equation (2.13), moves during the time-evolution of the system along the contour of the disk such that it remains the proximal point on the contour with respect to the table. The absolute velocity of point $C_{1}$ is obtained by differentiation of $\boldsymbol{r}_{O C_{1}}=\boldsymbol{r}_{O S}+\boldsymbol{r}_{S C_{1}}$,

$$
{ }_{B} \boldsymbol{v}_{C_{1}}=\underbrace{{ }_{B} \boldsymbol{v}_{S}+{ }_{B} \boldsymbol{\omega}_{I B} \times{ }_{B} \boldsymbol{r}_{S C_{1}}}_{{ }_{B} \boldsymbol{v}_{P_{1}}}+{ }_{B} \dot{\boldsymbol{r}}_{S C_{1}} .
$$

The term ${ }_{B} v_{P_{1}}$ represents the absolute velocity of the body-fixed point $P_{1}$, which at timeinstance $t$ coincides with point $C_{1}$. The second term, ${ }_{B} \dot{\boldsymbol{r}}_{S C_{1}}=-r{ }_{B} \dot{\boldsymbol{e}}_{z}^{K}$, corresponds to the velocity of point $C_{1}$ relative to the disk. For each nonhorizontal configuration of the $\operatorname{disk}\left(R_{33} \neq 1\right)$, the coordinate transformation $B=\left(\boldsymbol{e}_{x}^{B}, \boldsymbol{e}_{y}^{B}, \boldsymbol{e}_{z}^{B}\right) \mapsto K=\left(\boldsymbol{e}_{x}^{K}, \boldsymbol{e}_{y}^{K}, \boldsymbol{e}_{z}^{K}\right)$ is a rotation $\psi$ around axis $\boldsymbol{e}_{z}^{B}$. It therefore holds that ${ }_{B} \dot{\boldsymbol{e}}_{z}^{K}={ }_{B} \boldsymbol{\omega}_{B K} \times{ }_{B} \boldsymbol{e}_{z}^{K}=\dot{\psi}_{B} \boldsymbol{e}_{x}^{K}$, from which follows ${ }_{B} \dot{\boldsymbol{r}}_{S C_{1}}=-r \dot{\psi}{ }_{B} \boldsymbol{e}_{x}^{K}$. In addition, by differentiation of ${ }_{B} \boldsymbol{r}_{S C_{1}}$ (see (2.12) and (2.13)), we obtain

$$
{ }_{B} \dot{\boldsymbol{r}}_{S C_{1}}=-r \frac{\dot{R}_{32} R_{31}-\dot{R}_{31} R_{32}}{1-R_{33}^{2}}{ }_{B} \boldsymbol{e}_{x}^{K}
$$

By using the second equation in (2.6), we derive the following expression for $\dot{\psi}$ :

$$
\dot{\psi}=\frac{\dot{R}_{32} R_{31}-\dot{R}_{31} R_{32}}{1-R_{33}^{2}}=\left[\frac{R_{31} R_{33}}{1-R_{33}^{2}} \frac{R_{32} R_{33}}{1-R_{33}^{2}}-1\right]{ }_{B} \omega_{I B}
$$


We now introduce a number of velocity quantities for the relative kinematics of the interacting bodies. We first define the time-derivative of the gap function $g_{N 1}$. Using the notation $\gamma_{N 1}=\dot{g}_{N 1}$ with $g_{N 1}=\boldsymbol{e}_{z}^{I} \cdot \boldsymbol{r}_{C_{1}}$, and considering (2.16) and (2.17), it holds that

$$
\gamma_{N 1}=\boldsymbol{e}_{z}^{I} \cdot \boldsymbol{v}_{C_{1}}=\boldsymbol{e}_{z}^{I} \cdot \boldsymbol{v}_{P_{1}}=\dot{z}_{S}-\left({ }_{I} \boldsymbol{e}_{z}^{I}\right)^{\mathrm{T}}{ }_{I} \tilde{\boldsymbol{r}}_{S C_{1}} \boldsymbol{R}_{B} \boldsymbol{\omega}_{I B}
$$

That is to say, when $g_{N 1}=0, \gamma_{N 1}$ represents the relative velocity of the interacting bodies in the direction $\boldsymbol{e}_{z}^{I}$ normal to the contact plane $\left(\boldsymbol{e}_{x}^{I}, \boldsymbol{e}_{y}^{I}\right)$. Secondly, we define the tangential relative velocity $\gamma_{T 1}$ of the interacting bodies at contact point $C_{1}$ as a 2 -vector of which the elements are the first two components of ${ }_{I} v_{P_{1}}$,

$$
\gamma_{T 1}=\left[\begin{array}{c}
\dot{x}_{S}-\left({ }_{I} e_{x}^{I}\right)^{\mathrm{T}}{ }_{I} \tilde{\boldsymbol{r}}_{S C_{1}} \boldsymbol{R}_{B} \boldsymbol{\omega}_{I B} \\
\dot{y}_{S}-\left({ }_{I} \boldsymbol{e}_{y}^{I}\right)^{\mathrm{T}}{ }_{I} \tilde{\boldsymbol{r}}_{S C_{1}} \boldsymbol{R}_{B} \boldsymbol{\omega}_{I B}
\end{array}\right]
$$

The relative spin vector of the interacting bodies, $\omega_{\text {spin }}$, is a vector normal to the contact plane

$$
\boldsymbol{\omega}_{\text {spin }}=\omega_{\text {spin }} \boldsymbol{e}_{z}^{I}, \text { with } \omega_{\text {spin }}=\omega_{I B} \cdot \boldsymbol{e}_{z}^{I} .
$$

Furthermore, we introduce the spin velocity $\gamma_{\tau 1}$ as the product of the relative spin $\omega_{\text {spin }}$ and some quantity $\epsilon_{1}$,

$$
\gamma_{\tau 1}=\epsilon_{1} \omega_{\text {spin }}=\epsilon_{1}\left({ }_{I} e_{z}^{I}\right)^{\mathrm{T}}{ }_{I} \boldsymbol{\omega}_{I B},
$$

where $\epsilon_{1}$ is an assumed radius of a hypothetical circular contact area in the $\left(\boldsymbol{e}_{x}^{I}, \boldsymbol{e}_{y}^{I}\right)$-plane. Lastly, we define the relative "rolling" velocity $\gamma_{R}$ as the product of minus the radius of the disk and $\dot{\psi}(2.18)$,

$$
\gamma_{R 1}=-r \dot{\psi}=r\left[\begin{array}{lll}
\frac{-R_{31} R_{33}}{1-R_{33}^{2}} & \frac{-R_{32} R_{33}}{1-R_{33}^{2}} & 1
\end{array}\right]{ }_{B} \omega_{I B} .
$$

Subsequently, we treat the situation for which the disk, while being in contact, passes a horizontal singularity position at instant $t^{\star}$ and thereafter moves in the plane of the table $\left(g_{N 1}=g_{N 2}=g_{N 3}=0\right.$, and thus $\left.R_{33}=1\right)$. At such a time-instant $t^{\star}$, relations (2.13), (2.14) do not hold, and the points $C_{1}, C_{2}, C_{3}$ are defined by the continuity condition

$$
\boldsymbol{r}_{S C_{j}}^{+}\left(t^{\star}\right)=\boldsymbol{r}_{S C_{j}}^{-}\left(t^{\star}\right), \quad j=1,2,3,
$$

in which $\boldsymbol{r}_{S C_{j}}^{-}\left(t^{\star}\right)$ is considered to be known (an approximation will be given by the timestepping scheme; see Section 3). During the subsequent time-evolution (in the plane of the table), the three points $C_{1}, C_{2}, C_{3}$ are considered to be fixed to the disk. Consequently, the relative velocities $\gamma_{R j}, j=1,2,3$ are considered to be zero. According to the definition $g_{N j}=\boldsymbol{e}_{z}^{I} \cdot \boldsymbol{r}_{C_{j}}$, the time-derivative of the gap functions $\gamma_{N j}=\dot{g}_{N j}(j=1,2,3)$ are defined by expressions of the form (2.19). Similarly, $\boldsymbol{\gamma}_{T j}$ and $\gamma_{\tau j}(j=1,2,3)$ are defined by expressions of the form (2.20) and (2.22), respectively. 
Summarizing, for arbitrary configurations of the disk, we can write the relative velocities $\gamma_{N j}, \boldsymbol{\gamma}_{T j}, \gamma_{\tau j}$, and $\gamma_{R j}$, for $j=1,2,3$, as affine functions of $\boldsymbol{u}$,

$$
\begin{aligned}
& \gamma_{N j}=\boldsymbol{W}_{N j}^{\mathrm{T}} \boldsymbol{u}, \quad \boldsymbol{W}_{N j}=\left[\begin{array}{ll}
\left({ }_{I} \boldsymbol{e}_{z}^{I}\right)^{\mathrm{T}} \quad-\left({ }_{I} \boldsymbol{e}_{z}^{I}\right)^{\mathrm{T}}{ }_{I} \tilde{\boldsymbol{r}}_{S C_{j}} \boldsymbol{R}
\end{array}\right]^{\mathrm{T}}, \\
& \boldsymbol{\gamma}_{T j}=\boldsymbol{W}_{T j}^{\mathrm{T}} \boldsymbol{u}, \quad \boldsymbol{W}_{T j}=\left[\begin{array}{ll}
\left({ }_{I} \boldsymbol{e}_{x}^{I}\right)^{\mathrm{T}} & -\left({ }_{I} \boldsymbol{e}_{x}^{I}\right)^{\mathrm{T}}{ }_{I} \tilde{\boldsymbol{r}}_{S C_{j}} \boldsymbol{R} \\
\left({ }_{I} \boldsymbol{e}_{y}^{I}\right)^{\mathrm{T}} & -\left({ }_{I} \boldsymbol{e}_{y}^{I}\right)^{\mathrm{T}}{ }_{I} \tilde{\boldsymbol{r}}_{S C_{j}} \boldsymbol{R}
\end{array}\right]^{\mathrm{T}}, \\
& \gamma_{\tau j}=\boldsymbol{W}_{\tau j}^{\mathrm{T}} \boldsymbol{u}, \quad \boldsymbol{W}_{\tau j}=\left[\begin{array}{ll}
\mathbf{0} & \epsilon_{j}\left({ }_{I} \boldsymbol{e}_{z}^{I}\right)^{\mathrm{T}} \boldsymbol{R}
\end{array}\right]^{\mathrm{T}}, \\
& \gamma_{R j}=\boldsymbol{W}_{R j}^{\mathrm{T}} \boldsymbol{u}, \quad \boldsymbol{W}_{R 1}= \begin{cases}r\left[\begin{array}{lllll}
0 & 0 & 0 & \frac{-R_{31} R_{33}}{1-R_{33}^{2}} & \frac{-R_{32} R_{33}}{1-R_{33}^{2}} \\
1
\end{array}\right]^{\mathrm{T}}, & \text { only contact } 1 \text { active } \\
\mathbf{0}, & \text { else }\end{cases} \\
& \boldsymbol{W}_{R 2}=\boldsymbol{W}_{R 3}=\mathbf{0} \text {. }
\end{aligned}
$$

It can be demonstrated that $\boldsymbol{W}_{N 1}, \boldsymbol{W}_{N 2}, \boldsymbol{W}_{N 3}$ are three linearly independent vectors, which is of importance especially when dealing with noninterpenetration constraint violations (Section 3.3). Subsequently, we present the contact laws associated with the three unilateral constraints introduced above.

\subsection{Constitutive Laws}

Associated with each of the relative velocities $\boldsymbol{\gamma}$ in (2.25) are forces $\boldsymbol{\lambda}$ as their dual entities, for which we now introduce certain constitutive laws. These constitutive laws regard unilateral contact, Coulomb-Contensou friction, as well as rolling friction, and are formulated as set-valued interaction laws in the framework of convex analysis by applying the concept of the normal cone. The index $j$, used in the previous Section 2.2 to label the contact constraints, is omitted for brevity in the following.

2.3.1. Contact in Normal Direction: Signorini Condition. We assume Signorini's law to hold in the normal direction associated with a unilateral constraint

$$
g_{N} \geq 0, \quad \lambda_{N} \geq 0, \quad g_{N} \cdot \lambda_{N}=0,
$$

where $\lambda_{N}$ represents the normal contact force. Within the context of nonsmooth dynamics, we have to allow for temporal discontinuities, e.g., velocity jumps usually associated with collisions. The generalized velocity vector $\boldsymbol{u}$ is assumed to be a function of locally bounded variation on the time-interval $T$ of interest [17]. Such a function possesses a left limit $\boldsymbol{u}^{-}(t)$ and a right limit $\boldsymbol{u}^{+}(t)$ at every time-instance $t \in T$. Similarly, a left and right limit exist for the relative velocity $\gamma_{N}$ (2.25) considering $\boldsymbol{W}_{N}$ to be a continuous function of time. For a time-instance $t$ for which $g_{N}=0$ and $\lambda_{N}$ admits a right limit, it follows from (2.26) that

$$
\gamma_{N}^{+} \geq 0, \quad \lambda_{N}^{+} \geq 0, \quad \gamma_{N}^{+} \cdot \lambda_{N}^{+}=0,
$$


which is Signorini's condition on velocity level. This complementarity condition constitutes a contact law of a prospective type in the sense of Moreau. Therefore, it is possible to invoke the Viability Lemma [20] and to prove that if (2.27) is satisfied for almost every time-instance $t \in T$, and if $g_{N} \geq 0$ at the initial time-instance $t_{0}$, then the noninterpenetration of the interacting bodies is satisfied for all $t \in T$ succeeding $t_{0}$. Using concepts of convex analysis, we can write (2.27) in the form

$$
-\gamma_{N}^{+} \in N_{C_{N}}\left(\lambda_{N}^{+}\right)
$$

where $N_{C_{N}}\left(\lambda_{N}^{+}\right)$is the normal cone to $C_{N}$ at $\lambda_{N}^{+}$, with $C_{N}:=\mathbb{R}^{+}$.

2.3.2. Coulomb-Contensou Friction. Drilling friction is taken into account by using the Coulomb-Contensou friction model [13]. The Coulomb-Contensou friction model describes the coupled behaviour of spatial sliding friction and drilling friction. The tangential friction force $\boldsymbol{\lambda}_{T}$ and the normal drilling torque $\tau_{N}$ are derived from a nonsmooth scalar convex (pseudo) potential (dissipation function) expressed in terms of the sliding velocity $\boldsymbol{\gamma}_{T}$ and spin $\omega_{\text {spin. }}$. We assume that the disk locally deforms in the vicinity of a contact such that it has a circular contact surface with radius $\epsilon$ in the contact plane. Moreover, we consider a parabolic normal pressure distribution over the contact area. The Coulomb-Contensou friction model reads for a persistent contact as

$$
-\boldsymbol{\gamma}_{F} \in N_{C_{F}}\left(\boldsymbol{\lambda}_{F}\right),
$$

in which

$$
\boldsymbol{\lambda}_{F}=\left[\begin{array}{c}
\boldsymbol{\lambda}_{T} \\
\lambda_{\tau}
\end{array}\right] \text { with } \lambda_{\tau}=\frac{\tau_{N}}{\epsilon}, \quad \boldsymbol{\gamma}_{F}=\left[\begin{array}{l}
\boldsymbol{\gamma}_{T} \\
\gamma_{\tau}
\end{array}\right]=\boldsymbol{W}_{F}^{\mathrm{T}} \boldsymbol{u} \quad \text { with } \quad \boldsymbol{W}_{F}=\left[\begin{array}{ll}
\boldsymbol{W}_{T} & \boldsymbol{W}_{\tau}
\end{array}\right] .
$$

The convex set $C_{F}$, shown in Figure 3, is defined as follows:

$$
C_{F}:= \begin{cases}\left\{\lambda_{T}, \lambda_{\tau} \mid \frac{9}{64}\left(\frac{\eta}{\eta^{*}}\right)^{2}+\frac{9}{8}\left(\frac{\xi}{\xi^{*}}\right)^{2}-\frac{243}{1024}\left(\frac{\xi}{\xi^{*}}\right)^{4}\right. & \\ \left.-\frac{729}{32768}\left(\frac{\xi}{\xi^{*}}\right)^{6}+O\left(\left(\frac{\xi}{\xi^{*}}\right)^{8}\right) \leq 1\right\}, & \xi \leq \xi^{*} \\ \left\{\lambda_{T}, \lambda_{\tau} \mid \xi^{2}+5 \eta^{2}-\frac{75}{7} \eta^{4}+\frac{1250}{147} \eta^{6}+O\left(\eta^{8}\right) \leq 1\right\}, & \xi>\xi^{*}\end{cases}
$$

with

$$
\xi=\frac{\left\|\boldsymbol{\lambda}_{T}\right\|}{\mu_{F} \lambda_{N}}, \quad \eta=\frac{\lambda_{\tau}}{\mu_{F} \lambda_{N}}, \quad \xi^{*}=\frac{9}{32} \pi, \quad \eta^{*}=\frac{9}{128} \pi .
$$

The coefficient of friction in the Coulomb-Contensou friction model is denoted by $\mu_{F}$ and is in general a function of $\boldsymbol{\gamma}_{F}$. It is important to realize that the frictional sliding force $\lambda_{T}$ and the normalized drilling torque $\lambda_{\tau}$ are coupled by the Coulomb-Contensou friction law (2.29). If both the sliding velocity $\boldsymbol{\gamma}_{T}$ and spin $\omega_{\text {spin }}$ are identically zero, 


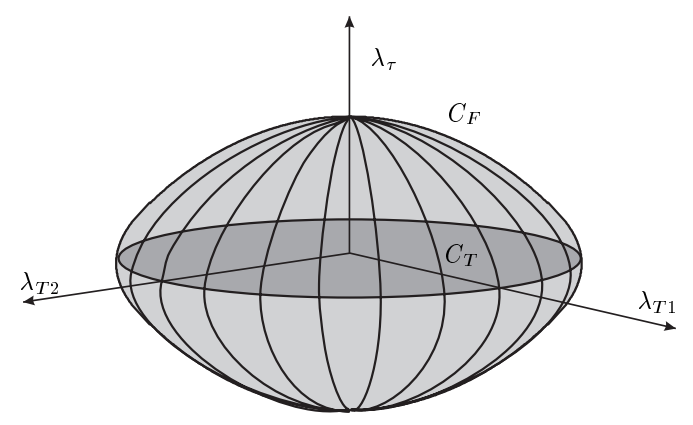

Fig. 3. Friction ball $C_{F}$.

then this case is referred to as "stick." If either the sliding velocity $\boldsymbol{\gamma}_{T}$ or the spin $\omega_{\text {spin }}$ is nonzero, then the contact is in "slip."

In the case of a persistent contact, the ratio $\left\|\boldsymbol{\gamma}_{T}\right\| / \mu_{F} \lambda_{N}$ is a strictly increasing smooth function of $\left\|\boldsymbol{\gamma}_{T}\right\| / \gamma_{\tau}$ for nonzero values of $\gamma_{\tau}=\epsilon \omega_{\text {spin }} \neq 0$. Similarly, the ratio $\lambda_{\tau} / \mu_{F} \lambda_{N}$ is a strictly increasing smooth function of the ratio $\gamma_{\tau} /\left\|\boldsymbol{\gamma}_{T}\right\|$ for nonzero values of $\boldsymbol{\gamma}_{T} \neq 0$. For example, this means that $\left\|\boldsymbol{\gamma}_{T}\right\|$ decreases when $\gamma_{\tau}$ increases for fixed values of $\boldsymbol{\gamma}_{T}$ and $\mu_{F} \lambda_{N}$. This physical effect can be demonstrated by an electric polishing machine used to clean floors. The machine is hard to move when the brushes are not rotating but can easily be pushed over the floor with rotating brushes.

2.3.3. Contour Friction. Classically, the resistance against rolling of two interacting bodies is modelled by Coulomb's law of rolling friction [23]. This set-valued force law relates a frictional couple transmitted by the contact to the relative velocity of rotation of the two interacting bodies. However, the classical rolling friction law is ambiguous and can lead to contradictions. Here, we will employ another type of rolling friction law, which models resistance against the movement of the contact point along the contour of the disk. We refer to this rolling friction law as the contour friction model throughout this paper. The contour friction model for a persistent contact relates the relative (rolling) velocity $\gamma_{R}$ to a force $\lambda_{R}$ opposing the movement of the contact point along the contour of the disk

$$
-\gamma_{R} \in N_{C_{R}}\left(\lambda_{R}\right)
$$

in which $C_{R}:=\left\{v \in \mathbb{R}|| v \mid \leq \mu_{R} \lambda_{N}\right\}$.

2.3.4. Constitutive Laws as Projections. Using the orthogonal projection operator proj, the force laws (2.28), (2.29), and (2.33) introduced above can be written in the equivalent form [1]:

$$
\boldsymbol{\lambda}_{X j}=\operatorname{proj}_{C_{X j}}\left(\boldsymbol{\lambda}_{X j}-\rho_{X} \boldsymbol{\gamma}_{X j}\right), \quad \rho_{X}>0 \quad \text { arbitrary, }
$$

for $X=N, F, R$ and $j=1,2,3$. 


\subsection{Nonsmooth Dynamics}

The absolute configuration of the system has been parameterized by the generalized coordinates

$$
\boldsymbol{q}=\left[\begin{array}{c}
{ }_{I} \boldsymbol{r}_{O S} \\
\boldsymbol{p}
\end{array}\right] \in \mathbb{R}^{7},
$$

as defined in Section 2.1. Let us consider a time-interval $T$ of the motion, not necessarily compact, of the form $T=\left[t_{0}, t_{e}\right]$ or $T=\left[t_{0}, \infty[\right.$. A motion of the system is described by a mapping $t \in T \mapsto \boldsymbol{q}(t) \in \mathbb{R}^{7}$, which is assumed to be locally absolutely continuous on $T$. Consequently, there exists a function $v: T \mapsto \mathbb{R}^{7}$, locally Lebesgue integrable, which permits us to obtain $\boldsymbol{q}$ by integration

$$
\forall t \in T, \quad \boldsymbol{q}(t)=\boldsymbol{q}\left(t_{0}\right)+\int_{t_{0}}^{t} \boldsymbol{v}(\sigma) d \sigma .
$$

The time-derivative of the Euler parameters $\boldsymbol{p}$ in terms of the angular velocity vector $\boldsymbol{\omega}$ is given by (2.9). The function $v \in \mathbb{R}^{7}$ is related to the generalized velocity vector $\boldsymbol{u} \in \mathbb{R}^{6}$ by the relation

$$
\boldsymbol{v}(t)=\boldsymbol{F}(\boldsymbol{q}(t)) \boldsymbol{u}(t), \quad \boldsymbol{F}=\left[\begin{array}{cc}
\boldsymbol{I}_{3} & \mathbf{0} \\
\mathbf{0} & \frac{1}{2} \overline{\boldsymbol{H}}^{\mathrm{T}}
\end{array}\right],
$$

where $\boldsymbol{F}$ is a continuous function of $\boldsymbol{q}$. In the context of smooth dynamics, we require the mapping $t \mapsto \boldsymbol{u}(t)$ to be locally absolutely continuous on $T$, which permits us to refer to its derivative $\dot{\boldsymbol{u}}(t)$. The time-evolution of the system is governed by the differential equation of smooth dynamics

$$
\boldsymbol{M}(\boldsymbol{q}) \dot{\boldsymbol{u}}-\boldsymbol{h}(\boldsymbol{q}, \boldsymbol{u})-\sum_{j} \boldsymbol{r}_{j}=\mathbf{0},
$$

with $\boldsymbol{M}=\operatorname{diag}\left(m \boldsymbol{I}_{3},{ }_{B} \boldsymbol{\Theta}_{S}\right),{ }_{B} \boldsymbol{\Theta}_{S}=\operatorname{diag}(A, A, C)$,

$$
\boldsymbol{h}=\left[\begin{array}{lllll}
0 & 0 & -m g & -{ }_{B} \tilde{\boldsymbol{\omega}}_{I B}{ }_{B} \boldsymbol{\Theta}_{S B} \boldsymbol{\omega}_{I B}
\end{array}\right]^{\mathrm{T}} .
$$

Due to the definition (2.7) of $\boldsymbol{u}$, the mass matrix $\boldsymbol{M} \in \mathbb{R}^{6 \times 6}$ of the system, being symmetric and positive definite, is diagonal and constant with respect to $\boldsymbol{q}$. The vector $\boldsymbol{h} \in \mathbb{R}^{6}$ contains all smooth forces/moments in the system, such as gyroscopic forces and moments and gravitation. The terms $\boldsymbol{r}_{j}, j=1,2,3$, represent the generalized components of the contact forces

$$
\boldsymbol{r}_{j}=\boldsymbol{W}_{j} \boldsymbol{\lambda}_{j}
$$

We have to consider the noninterpenetration constraints $\left(g_{N j}(\boldsymbol{q}) \geq 0(j=1,2,3)\right)$ and the constraint (2.1) on the Euler parameters together with the differential equation (2.38). Moreover, we have to add the constitutive laws for the unilateral frictional constraints defined in Section 2.3.

Within the context of nonsmooth dynamics (which we adopt), velocity jumps may occur, which are usually associated with collisions. The generalized velocity is considered to be a function $t \mapsto \boldsymbol{u}(t)$ of locally bounded variation on $T$ [17], i.e., $\boldsymbol{u}(t) \in \operatorname{lbv}\left(T, \mathbb{R}^{6}\right)$. 
The function $\boldsymbol{u}(t)$ therefore admits a left and right limit for all $t \in T$, and its derivative $\dot{\boldsymbol{u}}(t)$ exists for almost all $t \in T$ : The discontinuity points of $\boldsymbol{u}$ constitute an at most countable set being therefore Lesbesgue negligible.

However, the derivative $\dot{\boldsymbol{u}}$, pointwise defined, does not generally permit us to obtain $\boldsymbol{u}$ by Lebesgue integration. Instead, we use the Stieltjes (also called differential) measure $\mathrm{d} \boldsymbol{u}$. In this paper, we assume that the measure $\mathrm{d} \boldsymbol{u}$ can be decomposed in the form

$$
\mathrm{d} \boldsymbol{u}=\dot{\boldsymbol{u}} \mathrm{d} t+\left(\boldsymbol{u}^{+}-\boldsymbol{u}^{-}\right) \mathrm{d} \eta,
$$

in which $\mathrm{d} t$ denotes the Lebesgue measure and $\mathrm{d} \eta$ denotes the atomic measure. For each compact subinterval $\left[t_{1}, t_{2}\right]$ of $T$, it holds that

$$
\int_{\left[t_{1}, t_{2}\right]} \mathrm{d} \boldsymbol{u}=\boldsymbol{u}^{+}\left(t_{2}\right)-\boldsymbol{u}^{-}\left(t_{1}\right),
$$

which is even true for $t_{1}=t_{2}$,

$$
\int_{\left\{t_{1}\right\}} \mathrm{d} \boldsymbol{u}=\boldsymbol{u}^{+}\left(t_{1}\right)-\boldsymbol{u}^{-}\left(t_{1}\right),
$$

which is the velocity jump at $t_{1}$. The usual equation of motion, which relates acceleration to forces, is not suited to describe nonsmooth motion. We replace the equation of motion on acceleration level by an equality of measures in $\mathbb{R}^{6}$

$$
\boldsymbol{M}(\boldsymbol{q}) \mathrm{d} \boldsymbol{u}=\boldsymbol{h}(\boldsymbol{q}, \boldsymbol{u}) \mathrm{d} t+\sum_{j} \mathrm{~d} \boldsymbol{s}_{j},
$$

which holds on $T$. The contact efforts are not represented anymore by forces $\lambda_{j}$, but by contact impulsion measures. These contact impulsion measures are (for homogeneity reasons) denoted by $\mathrm{d} \boldsymbol{S}_{j}$, without paying attention to any function $\boldsymbol{S}_{j}$, admitting $\mathrm{d} \boldsymbol{S}_{j}$ as differential measure. The generalized components of $\mathrm{d} \boldsymbol{S}_{j}$ are defined by

$$
\mathrm{d} \boldsymbol{s}_{j}=\boldsymbol{W}_{j} \mathrm{~d} \boldsymbol{S}_{j} \in \mathbb{R}^{6}
$$

assuming $t \mapsto \boldsymbol{W}_{j}(\boldsymbol{q}(t))$ as continuous functions on $T$. Similar to the decomposition of $\mathrm{d} \boldsymbol{u}, \mathrm{d} \boldsymbol{S}_{j}$ is decomposed in

$$
\mathrm{d} \boldsymbol{S}_{j}=\boldsymbol{\lambda}_{j} \mathrm{~d} t+\boldsymbol{P}_{j} \mathrm{~d} \eta
$$

in which $\boldsymbol{\lambda}_{j}$ are the Lebesgue-measurable forces at contact point $C_{j}$ and $\boldsymbol{P}_{j}$ are the purely atomic impact impulsions (percussions). The nonsmooth equation of measures (2.43) allows us to formulate in a uniform manner the smooth and nonsmooth phases of motion. On each compact subinterval $\left[t_{1}, t_{2}\right]$ of $T$, the time-evolution of the generalized velocities $\boldsymbol{u}$ is governed by the following relation:

$$
\int_{\left[t_{1}, t_{2}\right]} \boldsymbol{M} \mathrm{d} \boldsymbol{u}=\int_{t_{1}}^{t_{2}} \boldsymbol{h} \mathrm{d} t+\int_{\left[t_{1}, t_{2}\right]} \sum_{j} \boldsymbol{W}_{j} \mathrm{~d} \boldsymbol{S}_{j},
$$

which is a balance of impulsions on the time-interval $\left[t_{1}, t_{2}\right]$. To this equation we have to add the constitutive laws associated with the unilateral frictional constraints. To be consistent with the formulation on impulse level, we state the constitutive laws between 
contact impulsions $\boldsymbol{\Lambda}_{j}=\int_{t_{1}}^{t_{2}} \mathrm{~d} \boldsymbol{S}_{j}$ and velocity quantities (see Section 3). The timeevolution of the generalized coordinates $\boldsymbol{q}$ can be obtained from (2.36). The generalized coordinates have to fulfill the noninterpenetration constraints $g_{N j}(\boldsymbol{q}) \geq 0,(j=1,2,3)$ and the constraint (2.1) on the Euler parameters.

\section{Numerical Algorithm: Time-Stepping Approach}

We now proceed to describe the numerical integration algorithm used for the simulations of Section 4. The numerical integration algorithm is a so-called time-stepping method [20], which is a time-discretization of the balance of impulsions (2.46).

\subsection{Time Discretization}

We discretize the time-interval $T$ of the motion in discrete times $t_{i}, i=1,2,3, \ldots$ with stepsize $\Delta t=t_{i+1}-t_{i}$. Consider a time-instance $t_{i}=t_{\mathrm{A}}$ at which the generalized coordinates $\boldsymbol{q}_{\mathrm{A}}$ and $\boldsymbol{u}_{\mathrm{A}}$ are given and which are admissible with respect to the unilateral constraints. We are interested in finding an approximant for the generalized coordinates and velocities at time $t_{\mathrm{E}}=t_{\mathrm{A}}+\Delta t$, respectively $\boldsymbol{q}_{\mathrm{E}}$ and $\boldsymbol{u}_{\mathrm{E}}$. The algorithm first estimates the generalized position at the midpoint $t_{\mathrm{M}}=t_{\mathrm{A}}+\frac{1}{2} \Delta t$ by the relation $\boldsymbol{q}_{\mathrm{M}}=\boldsymbol{q}_{\mathrm{A}}+$ $\frac{\Delta t}{2} \boldsymbol{F}\left(\boldsymbol{q}_{\mathrm{A}}\right) \boldsymbol{u}_{\mathrm{A}}$, with $\boldsymbol{F}$ defined by (2.37). The functions $\boldsymbol{W}_{j}(\boldsymbol{q}(t))$, assumed to be continuous on $T$, are approximated on the interval $\left[t_{\mathrm{A}}, t_{\mathrm{E}}\right]$ by the values $\boldsymbol{W}_{\mathrm{M} j}:=\boldsymbol{W}_{j}\left(\boldsymbol{q}_{\mathrm{M}}\right)$, which complies with the midpoint rule. The function $\boldsymbol{h}(\boldsymbol{q}, \boldsymbol{u})$ is approximated on the interval $\left[t_{\mathrm{A}}, t_{\mathrm{E}}\right]$ by the value $\boldsymbol{h}_{\mathrm{M}}:=\boldsymbol{h}\left(\boldsymbol{q}_{\mathrm{M}}, \boldsymbol{u}_{A}\right)$. Using the above approximants, the impulse balance (2.46) over the interval $\left[t_{\mathrm{A}}, t_{\mathrm{E}}\right]$ leads to the relation

$$
\boldsymbol{M}\left(\boldsymbol{u}_{\mathrm{E}}-\boldsymbol{u}_{\mathrm{A}}\right)=\boldsymbol{h}_{\mathrm{M}} \Delta t+\sum_{j \in I_{N}} \boldsymbol{W}_{\mathrm{M} j} \boldsymbol{\Lambda}_{j},
$$

in which $\boldsymbol{\Lambda}_{j}$ denotes the contact impulsion over the interval $\left[t_{\mathrm{A}}, t_{\mathrm{E}}\right]$ at contact point $C_{j}$. The summation of generalized contact impulses in (3.1) has to run only on the set of the contacts which are active on $\left[t_{\mathrm{A}}, t_{\mathrm{E}}\right]$. As an approximation, the set $I_{N}:=\left\{j \mid g_{N j}\left(\boldsymbol{q}_{\mathrm{M}}\right) \leq\right.$ $0\}$ is adopted. For each $j \in I_{N}$, we state the constitutive laws (2.34) between contact impulsions and velocity quantities in the general form

$$
\left(\boldsymbol{\Lambda}_{X}=\operatorname{proj}_{C_{X}}\left(\boldsymbol{\Lambda}_{X}-\rho_{X} \boldsymbol{\gamma}_{X \mathrm{E}}\right), \quad \rho_{X}>0 \quad \text { arbitrary }\right)_{j \in I_{N}},
$$

with

$$
\boldsymbol{\gamma}_{X \mathrm{E}}:=\boldsymbol{\gamma}_{X}\left(\boldsymbol{q}_{\mathrm{M}}, \boldsymbol{u}_{\mathrm{E}}\right)=\boldsymbol{W}_{X \mathrm{M}}^{\mathrm{T}} \boldsymbol{u}_{\mathrm{E}},
$$

for $X=N, F, R$ (see (2.25)), which corresponds in the case of an impact to a completely inelastic impact law. The frictional contact problem consists of the set of equations (3.1), (3.2), and (3.3), which is solved (see Section 3.2) for $\boldsymbol{u}_{\mathrm{E}}$ and the contact impulsions $\boldsymbol{\Lambda}_{j}$. The generalized coordinates at time $t_{E}$ are approximated by

$$
\boldsymbol{q}_{\mathrm{E}}=\boldsymbol{q}_{\mathrm{M}}+\frac{\Delta t}{2} \boldsymbol{F}\left(\boldsymbol{q}_{\mathrm{M}}\right) \boldsymbol{u}_{\mathrm{E}}
$$


We recognize in the integration scheme an implicit Euler rule for the generalized velocities and a midpoint rule for the generalized coordinates, which are, of course, coupled. The above integration scheme solves the contact problem using constitutive laws on velocity level. The unilateral constraints on position level as well as the unit norm constraint on the Euler parameters are in general not satisfied. A correction of the possible violations will be discussed in Section 3.3.

\subsection{Solving the Frictional Contact Problem}

In this subsection we discuss a numerical solution method for the frictional contact problem, which consists of the following set of equations:

$$
\begin{gathered}
\boldsymbol{M}\left(\boldsymbol{u}_{\mathrm{E}}-\boldsymbol{u}_{\mathrm{A}}\right)=\boldsymbol{h}_{\mathrm{M}} \Delta t+\sum_{j \in I_{N}} \boldsymbol{W}_{\mathrm{M} j} \boldsymbol{\Lambda}_{j}, \\
j \in I_{N}:\left(\begin{array}{c}
\Lambda_{N}=\operatorname{proj}_{C_{N}}\left(\Lambda_{N}-\rho_{N} \gamma_{N \mathrm{E}}\right) \\
\boldsymbol{\Lambda}_{F}=\operatorname{proj}_{C_{F}\left(\Lambda_{N}\right)}\left(\boldsymbol{\Lambda}_{F}-\rho_{F} \boldsymbol{\gamma}_{F \mathrm{E}}\right) \\
\Lambda_{R}=\operatorname{proj}_{C_{R}\left(\Lambda_{N}\right)}\left(\Lambda_{R}-\rho_{R} \gamma_{R \mathrm{E}}\right)
\end{array}\right)_{j},
\end{gathered}
$$

with

$$
\gamma_{N \mathrm{E}}=\boldsymbol{W}_{N \mathrm{M}}^{\mathrm{T}} \boldsymbol{u}_{\mathrm{E}}, \quad \boldsymbol{\gamma}_{F \mathrm{E}}=\boldsymbol{W}_{F \mathrm{M}}^{\mathrm{T}} \boldsymbol{u}_{\mathrm{E}}, \quad \gamma_{R \mathrm{E}}=\boldsymbol{W}_{R \mathrm{M}}^{\mathrm{T}} \boldsymbol{u}_{\mathrm{E}} .
$$

The nonlinear problem (3.5), (3.6) may be solved with various numerical techniques [20] [2]. Here, we proceed to briefly present a Modified Newton method [1] because of its simplicity. The Modified Newton algorithm consists of the following steps:

1. For $k=0$, give initial guesses for the contact impulsions: $\Lambda_{j}^{(0)}, \quad j \in I_{N}$;

2. Solve $\boldsymbol{u}_{\mathrm{E}}^{(k+1)}$ from $\boldsymbol{M}\left(\boldsymbol{u}_{\mathrm{E}}^{(k+1)}-\boldsymbol{u}_{\mathrm{A}}\right)=\boldsymbol{h}_{\mathrm{M}} \Delta t+\sum_{j \in I_{N}} \boldsymbol{W}_{\mathrm{M} j} \boldsymbol{\Lambda}_{j}^{(k)}$;

3. Project the impulses on unilateral frictional conditions: do the projections

$$
\begin{array}{cl}
\boldsymbol{\Lambda}_{N}^{(k+1)}=\operatorname{proj}_{C_{N}}\left(\boldsymbol{\Lambda}_{N}^{(k)}-\rho_{N} \boldsymbol{\gamma}_{N \mathrm{E}}^{(k+1)}\right), & \text { with } \boldsymbol{\gamma}_{N \mathrm{E}}^{(k+1)}=\boldsymbol{W}_{N \mathrm{M}}^{\mathrm{T}} \boldsymbol{u}_{\mathrm{E}}^{(k+1)}, \\
\boldsymbol{\Lambda}_{F}^{(k+1)}=\operatorname{proj}_{C_{F}\left(\Lambda_{N}\right)}\left(\boldsymbol{\Lambda}_{F}^{(k)}-\rho_{F} \boldsymbol{\gamma}_{F \mathrm{E}}^{(k+1)}\right), & \text { with } \boldsymbol{\gamma}_{F \mathrm{E}}^{(k+1)}=\boldsymbol{W}_{F \mathrm{M}}^{\mathrm{T}} \boldsymbol{u}_{\mathrm{E}}^{(k+1)}, \\
\boldsymbol{\Lambda}_{R}^{(k+1)}=\operatorname{proj}_{C_{R}\left(\Lambda_{N}\right)}\left(\boldsymbol{\Lambda}_{R}^{(k)}-\rho_{R} \boldsymbol{\gamma}_{R \mathrm{E}}^{(k+1)}\right), & \text { with } \boldsymbol{\gamma}_{R \mathrm{E}}^{(k+1)}=\boldsymbol{W}_{R \mathrm{M}}^{\mathrm{T}} \boldsymbol{u}_{\mathrm{E}}^{(k+1)}
\end{array}
$$

Repeat steps 2 and 3 until $\left\|\boldsymbol{\Lambda}_{N}^{(k+1)}-\boldsymbol{\Lambda}_{N}^{(k)}\right\|+\left\|\boldsymbol{\Lambda}_{F}^{(k+1)}-\boldsymbol{\Lambda}_{F}^{(k)}\right\|+\left\|\boldsymbol{\Lambda}_{R}^{(k+1)}-\boldsymbol{\Lambda}_{R}^{(k)}\right\|<\varepsilon$, where $\varepsilon$ is user-defined tolerance. On the first integration step, the Modified Newton iteration can start with $\boldsymbol{\Lambda}_{j}^{(0)}=\mathbf{0}$. For the subsequent integration steps, the value of the impulses calculated at the previous integration step is a good initial guess for the next step if no impact or stick-slip transition takes place. The choice of $\rho_{N}, \rho_{F}, \rho_{R}$ influences the rate of convergence of the Modified Newton algorithm. A general convergence, result of the Modified Newton algorithm does not exist. In practice, a small value usually results in a low convergence, and a large value may lead to divergence.

\subsection{Constraint Violation}

The time-stepping integration method solves the contact problem using constitutive laws on velocity level. The unilateral constraints on position level as well as the unit norm 
constraint on the Euler parameters, $g_{N j}(\boldsymbol{q}) \geq 0(j=1,2,3)$ and $\boldsymbol{p}^{\mathrm{T}} \boldsymbol{p}-1=0$, may not be satisfied at the end of the timestep $t=t_{\mathrm{E}}$. For this reason, the integration step is completed with a correction step of the possible violations. We introduce the set of violating contacts at time $t_{\mathrm{E}}, I_{V}:=\left\{j \mid g_{N \mathrm{E} j}<0\right\}$, and define the vector of violating gap function $\boldsymbol{g}_{V}=\left\{g_{N j}\right\}, j \in I_{V}$. The constraint violation functions are grouped in the vector $\boldsymbol{b}$ defined as follows:

$$
\boldsymbol{b}=\left[\begin{array}{c}
\boldsymbol{g}_{V} \\
\boldsymbol{p}^{\mathrm{T}} \boldsymbol{p}-1
\end{array}\right] .
$$

The correction step of the violations, which are supposed to be small, consists of replacing $\boldsymbol{q}_{\mathrm{E}}$ by its proximal point, called $\boldsymbol{q}_{C}$, in the set $\Phi:=\{\boldsymbol{q} \mid \boldsymbol{b}(\boldsymbol{q})=\mathbf{0}\}$. Proximity must be associated with a certain metric on $\mathbb{R}^{7}$. Let the matrix $\boldsymbol{A}(\boldsymbol{q}) \in \mathbb{R}^{7 \times 7}$, associated with a symmetric positive-definite bilinear form from $\mathbb{R}^{7} \times \mathbb{R}^{7}$ into $\mathbb{R}$, define such a metric. Assuming that the violations of the constraints are small compared to the accuracy of the integration scheme, and that the violations are of the same order of magnitude, we adopt for simplicity the metric defined by the standard scalar product of $\mathbb{R}^{7}$, and take $\boldsymbol{A}=\boldsymbol{I}_{7}$. The correction step of constraint violations consists of solving the following constrained optimization problem:

$$
\begin{array}{cc}
\text { minimize } & \frac{1}{2}\left\|\boldsymbol{x}-\boldsymbol{q}_{\mathrm{E}}\right\|^{2} \\
\text { subject to } & \boldsymbol{b}(\boldsymbol{x})=\mathbf{0} .
\end{array}
$$

Let $\mathcal{L}$ be the (ordinary) Lagrangian function associated with problem (3.9)

$$
\mathcal{L}(\boldsymbol{x}, \boldsymbol{\lambda})=\frac{1}{2}\left\|\boldsymbol{x}-\boldsymbol{q}_{\mathrm{E}}\right\|^{2}+\boldsymbol{\lambda}^{\mathrm{T}} \boldsymbol{b}(\boldsymbol{x}),
$$

in which $\boldsymbol{\lambda}$ represents the vector of Lagrange multipliers associated with the constraints. The stationarity conditions, which characterize a saddle point of $\mathcal{L}(\boldsymbol{x}, \boldsymbol{\lambda})$, can be written in the form

$$
\begin{aligned}
& \nabla_{x} \mathcal{L}(\boldsymbol{x}, \boldsymbol{\lambda})=\boldsymbol{x}-\boldsymbol{q}_{\mathrm{E}}+\partial \boldsymbol{b}^{\mathrm{T}}(\boldsymbol{x}) \boldsymbol{\lambda}=\mathbf{0}, \\
& \nabla_{\lambda} \mathcal{L}(\boldsymbol{x}, \boldsymbol{\lambda})=\boldsymbol{b}(\boldsymbol{x})=\mathbf{0},
\end{aligned}
$$

with

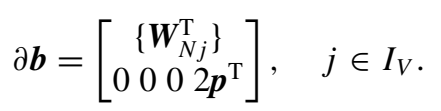

Thus, the problem consists of solving the set of nonlinear equations

$$
\begin{aligned}
& \boldsymbol{x}-\boldsymbol{q}_{\mathrm{E}}+\partial \boldsymbol{b}^{\mathrm{T}} \lambda=\mathbf{0} \\
& \boldsymbol{b}(\boldsymbol{x})=\mathbf{0}
\end{aligned} \Leftrightarrow \quad \boldsymbol{G}(\boldsymbol{x}, \boldsymbol{\lambda})=\mathbf{0},
$$

for the unknowns $(\boldsymbol{x}, \boldsymbol{\lambda})$. Defining $\boldsymbol{q}_{C}$ as a proximal point of $\boldsymbol{q}_{\mathrm{E}}$ on the set $\Phi$ with respect to the chosen metric means that the vector $\boldsymbol{q}_{C}-\boldsymbol{q}_{\mathrm{E}}$ is an element of the subspace of $\mathbb{R}^{7}$ generated by the linearly independent column vectors of the matrix $\partial \boldsymbol{b}^{\mathrm{T}}\left(\boldsymbol{q}_{C}\right)$. The value of $\partial \boldsymbol{b}^{\mathrm{T}}\left(\boldsymbol{q}_{C}\right)$ is assumed to be close to the value of $\partial \boldsymbol{b}^{\mathrm{T}}\left(\boldsymbol{q}_{\mathrm{E}}\right)$. It is therefore possible to 
solve the problem (3.13) by a quasi-Newton method for which the tangent operator $\partial \boldsymbol{G}$ is kept constant during the iterative process,

$$
\partial \boldsymbol{G}=\left[\begin{array}{cc}
\boldsymbol{I}_{7} & \partial \boldsymbol{b}^{\mathrm{T}}\left(\boldsymbol{q}_{\mathrm{E}}\right) \\
\partial \boldsymbol{b}\left(\boldsymbol{q}_{\mathrm{E}}\right) & \mathbf{0}
\end{array}\right] .
$$

\section{Numerical Results}

The numerical method described above has been used to simulate the time-evolution of a rolling disk. Four simulations, each associated with a different friction model and the same initial condition, have been conducted:

1. Simulation with only Coulomb friction: the Coulomb law is stated in the form (3.2), with $X=T$, and $C_{T}:=\left\{\boldsymbol{v} \in \mathbb{R}^{2} \mid\|\boldsymbol{v}\| \leq \mu_{T} \Lambda_{N}\right\}$;

2. Simulation with Coulomb and contour friction;

3. Simulation with Coulomb-Contensou friction;

4. Simulation with Coulomb-Contensou and contour friction.

The following dataset has been used for all simulations. Inertial properties: $m=0.3048$ $\mathrm{kg}, A=B=1.0716 \times 10^{-4} \mathrm{~kg} \mathrm{~m}^{2}, C=2.1433 \times 10^{-4} \mathrm{~kg} \mathrm{~m}^{2}, g=9.81 \mathrm{~m} / \mathrm{s}^{2}$. Geometrical properties: $r=3.75 \times 10^{-2} \mathrm{~m}$. Contact properties: $\mu_{T j}=0.3, \mu_{F j}=0.3$, $\epsilon_{j}=2 \times 10^{-3} \mathrm{~m}, \mu_{R j}=0.3 \times 10^{-3}$, for $j=1,2$, 3. Initially, the disk is considered to be in the plane $\left(O, \boldsymbol{e}_{x}^{I}, \boldsymbol{e}_{z}^{I}\right)$, which is orthogonal to the table. The disk interacts with the table through contact point $C_{1}$, which coincides with the origin $O$ of the reference frame $I$. The coordinate transformation $\left(\boldsymbol{e}_{x}^{I}, \boldsymbol{e}_{y}^{I}, \boldsymbol{e}_{z}^{I}\right) \mapsto\left(\boldsymbol{e}_{x}^{B}, \boldsymbol{e}_{y}^{B}, \boldsymbol{e}_{z}^{B}\right)$ can be accomplished by a finite rotation of an angle $\frac{\pi}{2}$ around the axis $\boldsymbol{e}_{x}^{I}$. Consequently, it holds that

$$
\boldsymbol{q}_{0}=\boldsymbol{q}\left(t_{0}\right)=\left[\begin{array}{lllllll}
0 & 0 & r & \cos \frac{\pi}{4} & \sin \frac{\pi}{4} & 0 & 0
\end{array}\right]^{\mathrm{T}} .
$$

At time-instant $t_{0}$, the disk is rolling without sliding on the table. The angular velocity of the disk is taken as a vector in the horizontal plane, more precisely, ${ }_{B} \omega_{I B}\left(t_{0}\right)=$ $\|\boldsymbol{\omega}\|[\sin \delta 0 \cos \delta]^{\mathrm{T}}$ with $\|\boldsymbol{\omega}\|=10 \mathrm{rad} / \mathrm{s}$ and $\delta=0.035 \mathrm{rad}$. The sticking condition at the initial time-instant leads to a direct relation between the velocity of the centre of mass $S$ and the angular velocity $\boldsymbol{\omega}$, i.e., ${ }_{I} \dot{\boldsymbol{r}}_{S}\left(t_{0}\right)=-r\|\boldsymbol{\omega}\|[\cos \delta \sin \delta 0]^{\mathrm{T}}$. Consequently the initial velocity of the system $\boldsymbol{u}_{0}=\boldsymbol{u}\left(t_{0}\right)$ reads as

$$
\boldsymbol{u}_{0}=\|\boldsymbol{\omega}\|\left[\begin{array}{lllllll}
-r & \cos \delta & -r \sin \delta & 0 & \sin \delta & 0 & \cos \delta
\end{array}\right]^{\mathrm{T}} .
$$

The results of the four simulations are presented in a condensed form in the following subsections together with some brief comments. The stepsize of the numerical scheme is given the fixed value $\Delta t=10^{-4} \mathrm{~s}$ for all simulations.

\subsection{Simulation with Coulomb Friction}

The results of the simulation with Coulomb friction (i.e., no Contensou or contour friction) are presented in Figure 4. The graph $\left(x_{S}, y_{S}\right)$ in the left column describes the 

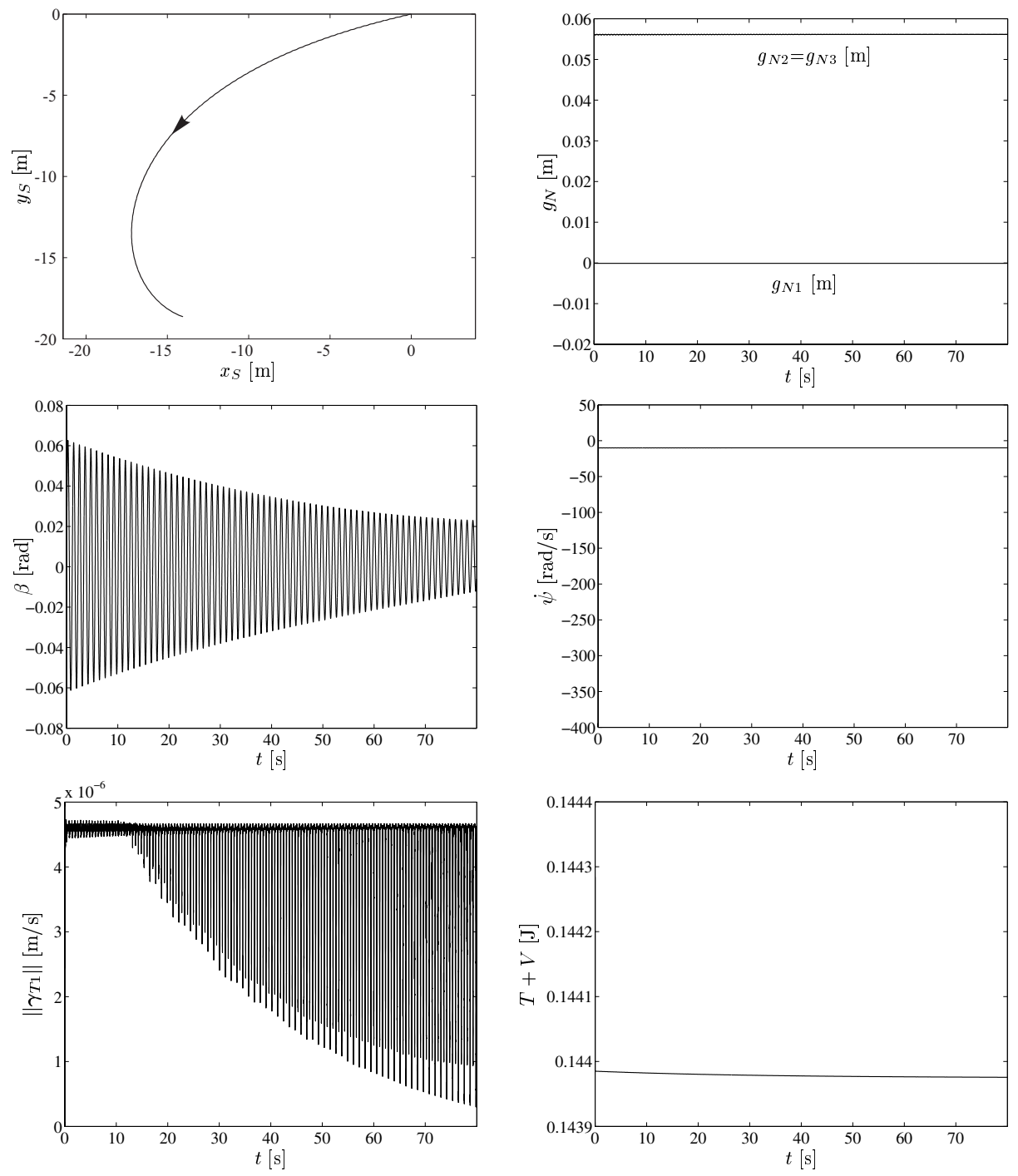

Fig. 4. Simulation with Coulomb friction.

trajectory of the centre of mass $S$ during the first 80 seconds of the motion. According to graphs $\left(t, g_{N}\right)$ and $\left(t,\left\|\boldsymbol{\gamma}_{T 1}\right\|\right)$, contact 1 , which is the only active contact, remains in a sticking state during the whole time-evolution. The motion of the disk is therefore a pure rolling motion; thus, Coulomb friction acts as an anholonomic constraint. The graph $(t, \dot{\psi})$ is representative for the relative kinematics of the contact point with respect to the disk: The magnitude $\dot{\psi}$, given by (2.18), defines the angular velocity of the contact point $C_{1}$ in its motion along the contour of the disk. It appears that this velocity remains (quasi) constant and equal to the value $\left\|\boldsymbol{\omega}\left(t_{0}\right)\right\|$. The graph $(t, \beta), \beta$ being the angle of 
nutation (Figure 8), shows that the rolling motion of the disk is accompanied by small oscillations around the global motion of the disk. The pure rolling condition causes the mechanical system under consideration to be conservative, as no dissipation due to sliding can occur. However, the graph $(t, T+V)$ shows a decrease in the total mechanical energy of the system. During the time-interval under consideration with $\Delta t=10^{-4} \mathrm{~s}$, the numerical scheme dissipates $0.2 \%$ of the initial system energy. The numerical results show the efficiency of the correction step of possible constraint violations, which has been presented in Section 3.3 (see for example graph $\left(t, g_{N}\right)$ ).

\subsection{Simulation with Coulomb and Contour Friction}

The results of the simulation with Coulomb and contour friction (i.e., no Contensou friction) are presented in Figure 5. During the first $25 \mathrm{~s}$ of the time-evolution, the disk rolls without sliding over the table. A part of the initial energy of the system is dissipated due to the presence of contour friction during this time-interval, as illustrated in the graph $(t, T+V)$. Subsequently, when the inclination of the disk reaches approximately the value $\beta=0.45 \mathrm{rad}$, the disk slides laterally over the table during $0.5 \mathrm{~s}$. The peak that can be observed in the graph $\left(t,\left\|\boldsymbol{\gamma}_{T 1}\right\|\right)$ shows the large variations of the sliding velocity on the corresponding short time-interval. The sliding motion causes a considerable decrease in the inclination and in the total energy. At the same time, the angular velocity $\dot{\psi}$ of the contact point $C_{1}$ with respect to the disk increases. During the subsequent period $25.5 \mathrm{~s}<t<67 \mathrm{~s}$, two distinct phases of motion can be identified. First, a phase of sticking takes place on the time-interval $25.5 \mathrm{~s}<t<32 \mathrm{~s}$. During this period there is a transfer from kinetic energy to potential energy. The inclination of the disk with respect to the table increases (the disk becomes more upright), as shown in the graphs $\left(t, g_{N}\right)$ and $(t, \beta)$, whereas $\dot{\psi}$ decreases. On the following time-interval $32 \mathrm{~s}<t<67 \mathrm{~s}$, contact 1 switches successively between sticking and sliding states. The potential energy of the system decreases and the velocity of the contact point $C_{1}$ with respect to the disk increases as the disk falls down. The angular velocity $\dot{\psi}$ of $C_{1}$ tends to infinity when the disk approaches a horizontal configuration, and contour friction becomes the main mechanism of energy dissipation. At the same time the angular velocity $\boldsymbol{\omega}_{I B}$ of the disk tends to zero. At time $t=67 \mathrm{~s}$, as contact 2 and 3 become active, the disk reaches a horizontal equilibrium configuration. Parts of various graphs are indiscernible due to high-frequency oscillations, which we discuss in Section 6.

\subsection{Simulation with Coulomb-Contensou Friction}

The results of the simulation with Coulomb-Contensou friction (i.e., no contour friction) are presented in Figure 6. Immediately after $t=0 \mathrm{~s}$, the disk starts to slide laterally (and also to pivot), which leads to a decrease in the inclination of the disk and therefore in the potential energy of the system (see graphs $\left(t, g_{N}\right)$ and $\left.(t, \beta)\right)$. At time-instant $t=0.4 \mathrm{~s}$, when the nutation reaches the value $\beta=0.45 \mathrm{rad}$, the sliding velocity of contact 1 highly increases and subsequently decreases on a short time-interval, as is illustrated by the peak in the graph $\left(t,\left\|\boldsymbol{\gamma}_{T 1}\right\|\right)$. A high decrease in the friction torque $\tau_{N}$ can be observed on this short time-interval, which is in agreement with the Coulomb- 

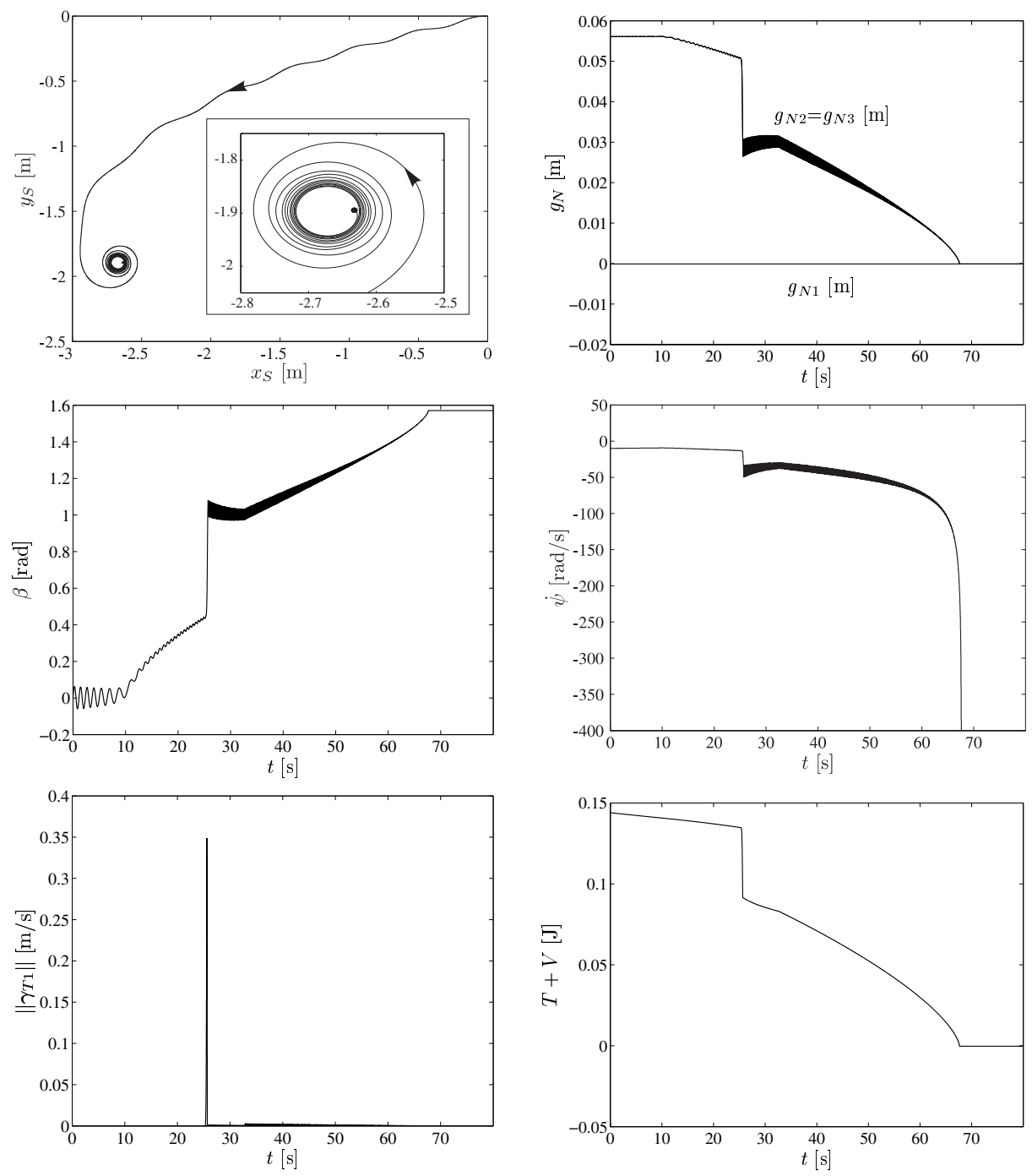

Fig. 5. Simulation with Coulomb and contour friction.

Contensou friction model (see Figure 6b in [13]). Consequently, the dissipated energy is mostly due to the work done by the tangential contact force $\boldsymbol{\lambda}_{T_{1}}$. It is to be noted that in the previous case (simulation with Coulomb and contour friction) the rapid sliding phenomenon happens for the same value of $\beta$, but at a much later time-instant $t=25 \mathrm{~s}$. During the subsequent part of the motion, in the presence of sliding and spinning, the rate of dissipation diminishes as the spin of the disk reduces. The total energy of the system seems to decrease asymptotically to zero. For an asymptotical decrease to zero, the disk cannot reach a horizontal equilibrium configuration in finite time but only approaches this equilibrium when time tends to infinity. 

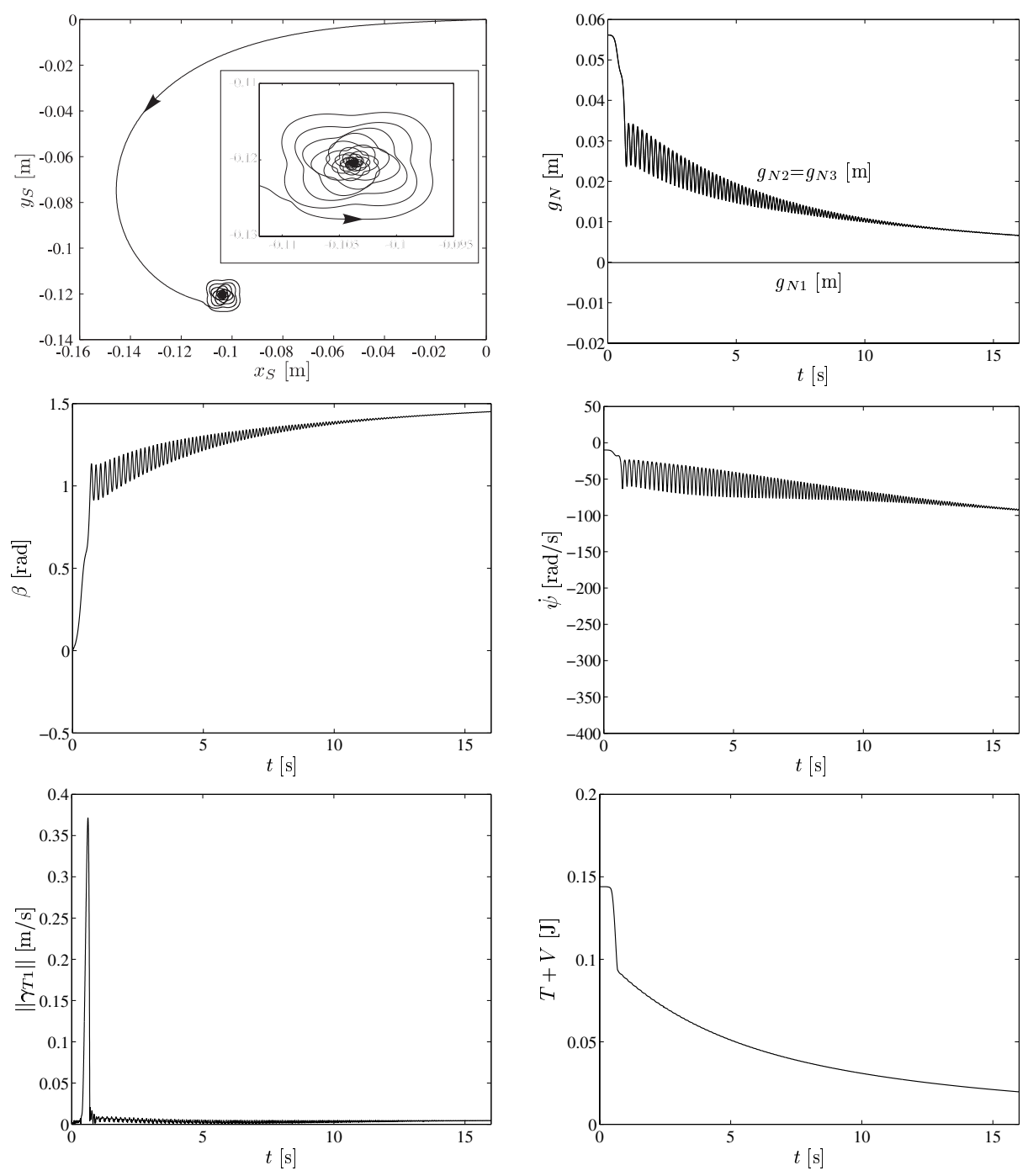

Fig. 6. Simulation with Coulomb-Contensou friction.

\subsection{Simulation with Coulomb-Contensou and Contour Friction}

The results of the simulation with Coulomb-Contensou and contour friction are presented in Figure 7. The first part of the motion, including the peak in the sliding velocity (see graph $\left(t,\left\|\boldsymbol{\gamma}_{T 1}\right\|\right)$ ), is similar to the time-evolution calculated on this time-interval with Coulomb-Contensou friction. The contour friction force $\lambda_{R 1}$ is very small because the contour friction coefficient $\mu_{R 1}$ is taken to be a small constant $3 \cdot 10^{-4}$. Meanwhile, the contour rolling velocity $\gamma_{R 1}$ is rather small during this part of motion. The dissipation due to contour friction is therefore small with respect to the dissipation due to 

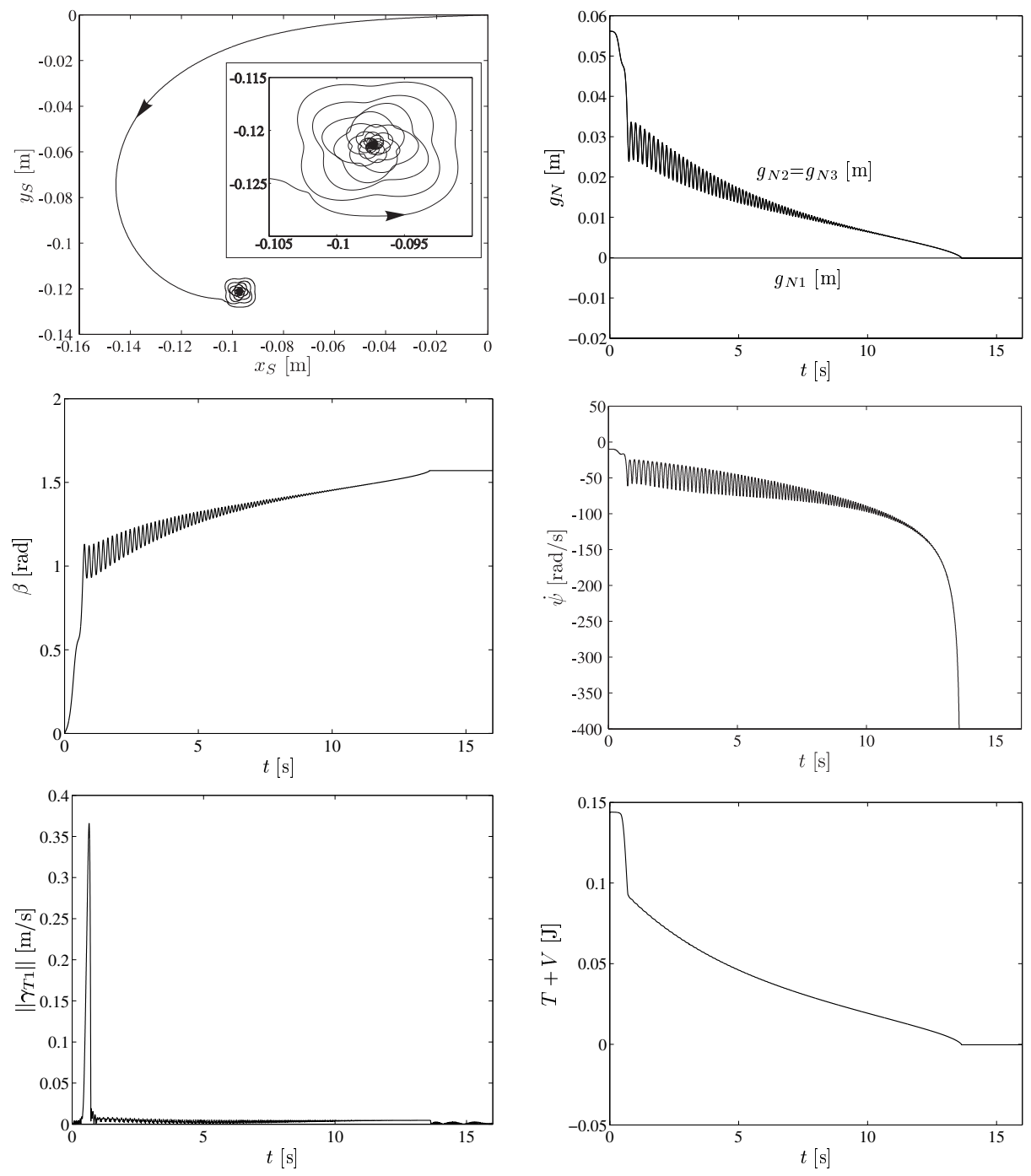

Fig. 7. Simulation with Coulomb-Contensou and contour friction.

Coulomb-Contensou friction. As for the previous case (Coulomb-Contensou friction), the dissipated energy is mostly due to the work done by the tangential contact force. During the subsequent part of the motion, in the presence of sliding and spinning, the inclination of the disk with respect to the table and its angular velocity fall down, whereas the velocity of the contact point with respect to the disk $C_{1}$ is strongly increasing (see graphs $(t, \beta)$ and $(t, \dot{\psi}))$. When the disk approaches a horizontal configuration, the contour rolling velocity $\gamma_{R 1}$ tends to infinity, and contour friction becomes the main mechanism of energy dissipation. At time $\mathrm{t}=13.6 \mathrm{~s}$, as contacts 2 and 3 become active, 


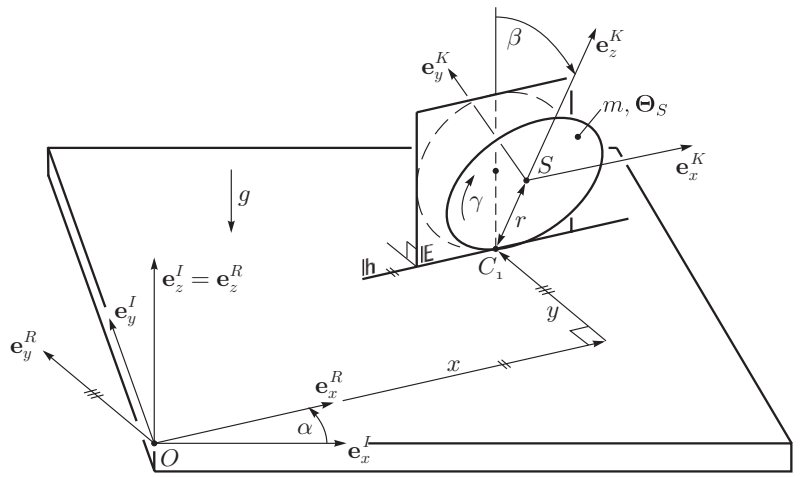

(c)

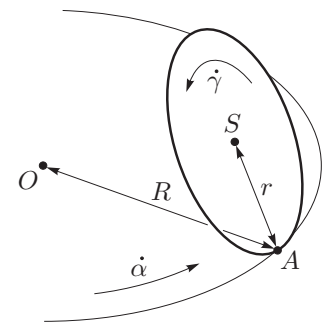

(d)

Fig. 8. Analytical analysis: (a) parametrization of the disk, (b) circular rolling motion.

the disk reaches a horizontal equilibrium configuration. Contour friction plays a crucial role in the end phase of the motion and causes the disk to reach a horizontal equilibrium configuration in a finite time.

\section{Analytical Analysis of Rolling Motion}

The simulations of the previous section reveal various dynamic effects. The consideration of an analytical model of the pure rolling motion of the disk permits us to give an interpretation of these dynamic effects and therefore contributes to a better understanding of the dynamics of a rolling disk.

\subsection{Analytical Model}

The analytical model, presented here, describes the mechanical system under consideration as a disk submitted to a bilateral contact constraint and a sticking condition at contact point $C_{1}$. We define a parametrization of the disk $(x, y, \alpha, \beta, \gamma)$ as illustrated in Figure 8a, which accounts for the bilateral contact constraint in normal direction. The sticking condition at contact point $C_{1}$ can be expressed by means of the two nonholonomic scalar constraints

$$
\dot{x}-y \dot{\alpha}-r \dot{\gamma}=0 \quad \text { and } \quad \dot{y}+x \dot{\alpha}=0 .
$$

The conditions (5.1) permit us to express $\dot{x}$ and $\dot{y}$ as functions of $\dot{\alpha}, \dot{\beta}$, and $\dot{\gamma}$. Subsequently, we write the equations of motion using the coordinates $(x, y, \alpha, \beta, \gamma)$ and minimal velocities $(\dot{\alpha}, \dot{\beta}, \dot{\gamma})$. 
First, we derive the angular velocity ${ }_{K} \boldsymbol{\omega}_{I B}$ and the velocity of the center of mass ${ }_{K} \boldsymbol{v}_{S}$ of the disk, by using frame $K=\left(\boldsymbol{e}_{x}^{K}, \boldsymbol{e}_{y}^{K}, \boldsymbol{e}_{z}^{K}\right)$,

$$
\begin{aligned}
{ }_{K} \boldsymbol{\omega}_{I B} & =\dot{\alpha}_{K} \boldsymbol{e}_{z}^{R}+\dot{\beta}{ }_{K} \boldsymbol{e}_{x}^{K}+\dot{\gamma}_{K} \boldsymbol{e}_{y}^{K} \quad{ }_{K} \boldsymbol{v}_{S}={ }_{K} \boldsymbol{\omega}_{I B} \times{ }_{K} \boldsymbol{r}_{C_{1} S} \\
& =\underbrace{\left[\begin{array}{ccc}
0 & 1 & 0 \\
\sin \beta & 0 & 1 \\
\cos \beta & 0 & 0
\end{array}\right]}_{{ }_{K} \bar{J}_{R}}\left[\begin{array}{c}
\dot{\alpha} \\
\dot{\beta} \\
\dot{\gamma}
\end{array}\right], \quad=\underbrace{\left[\begin{array}{ccc}
r \sin \beta & 0 & r \\
0 & -r & 0 \\
0 & 0 & 0
\end{array}\right]}_{{ }_{K} \bar{J}_{S}}\left[\begin{array}{c}
\dot{\alpha} \\
\dot{\beta} \\
\dot{\gamma}
\end{array}\right] .
\end{aligned}
$$

Note that frame $K$ is not body-fixed, but moves along with the disk such that the $\boldsymbol{e}_{x}^{K}$-axis remains parallel to the table.

Furthermore, we introduce the linear momentum ${ }_{K} \overline{\boldsymbol{p}}=m_{K} \boldsymbol{v}_{S}$ and angular momentum ${ }_{K} \overline{\boldsymbol{N}}_{S}={ }_{K} \boldsymbol{\Theta}_{S K} \boldsymbol{\omega}_{I B}$ of the disk. Considering the parameters for the disk $A=B=\frac{m r^{2}}{4}$ and $C=\frac{m r^{2}}{2}$, it holds that

$$
\begin{gathered}
{ }_{K}(\dot{\overline{\boldsymbol{p}}})=m r\left[\begin{array}{c}
\ddot{\alpha} \sin \beta+\ddot{\gamma}+2 \dot{\alpha} \dot{\beta} \cos \beta \\
-\ddot{\beta}+\dot{\alpha}^{2} \sin \beta \cos \beta+\dot{\alpha} \dot{\gamma} \cos \beta \\
-\dot{\beta}^{2}-\dot{\alpha}^{2} \sin ^{2} \beta-\dot{\alpha} \dot{\gamma} \sin \beta
\end{array}\right], \\
{ }_{K}\left(\dot{\overline{\boldsymbol{N}}}_{S}\right)=\frac{1}{4} m r^{2}\left[\begin{array}{c}
\ddot{\beta}-\dot{\alpha}^{2} \sin \beta \cos \beta-2 \dot{\alpha} \dot{\gamma} \cos \beta \\
2 \ddot{\alpha} \sin \beta+2 \ddot{\gamma}+2 \dot{\alpha} \dot{\gamma} \\
\ddot{\alpha} \cos \beta+2 \beta \dot{\gamma}
\end{array}\right] .
\end{gathered}
$$

Subsequently, we apply the principle of virtual power for virtual velocities that are compatible with the sticking condition. As the constraints are considered to be ideal, only the action of gravity arises in the virtual power of external effort. The action of the gravity on the disk is described by a force ${ }_{K} \boldsymbol{F}=m \tilde{g} r[0-\sin \beta-\cos \beta]^{\mathrm{T}}$ and a momentum ${ }_{K} \boldsymbol{M}_{S}=\mathbf{0}$ with $\tilde{g}=\frac{g}{r}$. From the principle of virtual power, follow the projected Newton-Euler equations [8],

$$
{ }_{K} \overline{\boldsymbol{J}}_{S}^{\mathrm{T}}\left[{ }_{K}(\dot{\overline{\boldsymbol{p}}})-{ }_{K} \boldsymbol{F}\right]+{ }_{K} \overline{\boldsymbol{J}}_{R}^{\mathrm{T}}\left[{ }_{K}\left(\dot{\overline{\boldsymbol{N}}}_{S}\right)-{ }_{K} \boldsymbol{M}_{S}\right]=\mathbf{0},
$$

which yield the equations of motion of the disk:

$$
\begin{aligned}
\left(5 \sin ^{2} \beta+1\right) \ddot{\alpha}+6 \ddot{\gamma} \sin \beta+10 \dot{\alpha} \dot{\beta} \sin \beta \cos \beta+2 \dot{\beta} \dot{\gamma} \cos \beta & =0, \\
5 \ddot{\beta}-5 \dot{\alpha}^{2} \sin \beta \cos \beta-6 \dot{\alpha} \dot{\gamma} \cos \beta-4 \tilde{g} \sin \beta & =0, \\
6 \ddot{\alpha} \sin \beta+6 \ddot{\gamma}+10 \dot{\alpha} \dot{\beta} \cos \beta & =0 .
\end{aligned}
$$

\subsection{Circular Rolling Motion}

We now proceed to analyze a particular type of rolling motion. We consider the type of motion $\left(x_{0}(t), y_{0}(t), \alpha_{0}(t), \beta_{0}(t), \gamma_{0}(t)\right)$ for which $x_{0}=0$ and $\beta_{0}=$ const. $\left(0<\beta_{0}<\frac{\pi}{2}\right)$ in time. It follows that $\dot{x}_{0}=\ddot{x}_{0}=0$ and $\dot{\beta}_{0}=\ddot{\beta}_{0}=0$. Consequently, the sticking conditions (5.1) yield

$$
\begin{aligned}
& \dot{y}_{0}=-x_{0} \dot{\alpha}_{0}=0 \Rightarrow \dot{y}_{0}=\ddot{y}_{0}=0 ; y_{0}=\text { const. }=R, \\
& \dot{x}_{0}=y_{0} \dot{\alpha}_{0}+r \dot{\gamma}_{0} \Rightarrow 0=R \dot{\alpha}_{0}+r \dot{\gamma}_{0} .
\end{aligned}
$$


During such a motion, the inclination of the disk $\beta_{0}$ with respect to the vertical $\boldsymbol{e}_{z}^{I}$ and the height of the center of mass are constant in time. As contact point $C_{1}$ moves on the contour of the disk (relative motion), it describes on the table (absolute motion) a circular trajectory $(O, R)$ of radius $R$ around the origin $O$ of the inertial frame (see Figure 8). Equation (5.9) is the condition for pure rolling, which means that, for a given time-interval of the motion, the arc lengths covered by the contact point $C_{1}$ on both the perimeter of the circle $(O, R)$ and the perimeter of the disk are equal. In the following we refer to such motion as circular rolling motion. Subtraction of (5.7). $\sin \beta_{0}$ from (5.5) yields, together with $\dot{\beta}_{0}=0$, to $\ddot{\alpha}_{0}=0$. Consequently, it can be deduced from the pure rolling condition (5.9) that both $\dot{\alpha}_{0}$ and $\dot{\gamma}_{0}$ are constant in time for circular rolling motion. In the following we define $\rho$ (see Figure 8 ) as

$$
\rho=\frac{r}{R} .
$$

It follows from the equation of motion (5.6) and the pure rolling condition (5.9) written as

$$
\dot{\alpha}_{0}=-\rho \dot{\gamma}_{0}
$$

that

$$
\dot{\gamma}_{0}^{2}=\frac{4 \tilde{g} \sin \beta_{0}}{\left(6-5 \rho \sin \beta_{0}\right) \rho \cos \beta_{0}}, \quad \text { with } \quad 0<\rho<\frac{6}{5 \sin \beta_{0}} .
$$

Subsequently, we study a particular type of circular rolling motion for which, as the disk is rolling on the table, the center of mass $S$ remains on the axis $\left(O, \boldsymbol{e}_{z}^{I}\right)$. This type of motion is characterized by

$$
r \sin \beta_{0}=R \quad \Rightarrow \quad \rho \sin \beta_{0}=1,
$$

which fulfills the restriction in (5.12). In this case $\dot{\gamma}^{2}$ and $\dot{\alpha}^{2}$ can be written as

$$
\dot{\gamma}_{0}^{2}=\frac{4 \tilde{g}}{\rho \sqrt{\rho^{2}-1}} \quad \text { and } \quad \dot{\alpha}_{0}^{2}=\rho^{2} \dot{\gamma}_{0}^{2}=\frac{4 \tilde{g} \rho}{\sqrt{\rho^{2}-1}} .
$$

Considering (5.2) together with (5.11), (5.13), and (5.14) we deduce

$$
\left\|\omega_{I B}\right\|^{2}=4 \tilde{g} \sqrt{1-\frac{1}{\rho^{2}}} \quad \text { and } \quad \boldsymbol{v}_{S}=\mathbf{0},
$$

which reveals that the center of mass $S$ is immobile with respect to the inertial frame. We call this type of motion stationary rolling motion. In the limit of $\beta_{0} \rightarrow \frac{\pi}{2}$, it holds that $\rho \rightarrow 1$. Consequently, it follows that $\dot{\alpha}_{0}^{2}$ and $\dot{\gamma}_{0}^{2} \rightarrow+\infty$ while $\boldsymbol{\omega}_{I B} \rightarrow \mathbf{0}$. The contact point $C_{1}$ therefore moves infinitely fast on the circle $(O, R)$ with radius $R \rightarrow r$, and moves infinitely fast on the contour of the disk, while the disk practically does not rotate.

\subsection{Small Oscillations around Circular Rolling Motion}

In this subsection we study small oscillations around a circular rolling motion using a harmonic balance method. We consider the motion to be composed of the circular rolling 
motion with superimposed harmonic terms of frequency $\omega$,

$$
\begin{aligned}
\dot{\alpha}=\dot{\alpha}_{0}+a \cos \omega t, & \dot{\alpha}_{0}=\text { const }, \\
\beta=\beta_{0}+b \cos \omega t, & \beta_{0}=\text { const }, \\
\dot{\gamma}=\dot{\gamma}_{0}+c \cos \omega t, & \dot{\gamma}_{0}=\text { const },
\end{aligned}
$$

and with small amplitudes $a \ll 1, b \ll 1, c \ll 1$. Substitution of the Ansatz (5.16) in the equation of motion (5.5) gives

$$
\begin{aligned}
& -\left(5 \sin ^{2}\left(\beta_{0}+b \cos \omega t\right)+1\right) a \omega \sin \omega t-6 \omega c \sin \omega t \sin \left(\beta_{0}+b \cos \omega t\right) \\
& -10\left(\dot{\alpha}_{0}+a \cos \omega t\right) \omega b \sin \omega t \sin \left(\beta_{0}+b \cos \omega t\right) \cos \left(\beta_{0}+b \cos \omega t\right) \\
& -2 \omega b\left(\dot{\gamma}_{0}+c \cos \omega t\right) \sin \omega t \cos \left(\beta_{0}+b \cos \omega t\right)=0 .
\end{aligned}
$$

Subsequently, we equate first-order terms in $a, b$, and $c$ and divide by $-\omega \sin \omega t$

$$
\left(5 \sin ^{2} \beta_{0}+1\right) a+6 c \sin \beta_{0}+10 \dot{\alpha}_{0} b \sin \beta_{0} \cos \beta_{0}+2 b \dot{\gamma}_{0} \cos \beta_{0}=0 .
$$

Similarly, we substitute (5.16) in (5.6) and equate the first-order terms

$$
\begin{aligned}
b \omega^{2}= & -2 \dot{\alpha}_{0} a \sin \beta_{0} \cos \beta_{0}+\dot{\alpha}_{0}^{2} b\left(\sin ^{2} \beta_{0}-\cos ^{2} \beta_{0}\right) \\
& -\frac{6}{5}\left(a \dot{\gamma}_{0}+c \dot{\alpha}_{0}\right) \cos \beta_{0}+\frac{6}{5} \dot{\alpha}_{0} \dot{\gamma}_{0} b \sin \beta_{0}-\frac{4}{5} \tilde{g} b \cos \beta_{0} .
\end{aligned}
$$

Considering (5.16) together with (5.7) and equating first-order terms yields

$$
a \sin \beta_{0}+c+\frac{5}{3} \dot{\alpha}_{0} b \cos \beta_{0}=0 .
$$

We now subtract (5.19). $6 \sin \beta_{0}$ from (5.17)

$$
a \cos ^{2} \beta_{0}+2 b \dot{\gamma_{0}} \cos \beta_{0}=0 \Rightarrow a=-\frac{2 b \dot{\gamma}_{0}}{\cos \beta_{0}},
$$

which gives the amplitude $a$ as a function of $b$. Using (5.19) we find the amplitude $c$ as a function of $b$,

$$
c=\frac{2 b \dot{\gamma}_{0}}{\cos \beta_{0}} \sin \beta_{0}-\frac{5}{3} \dot{\alpha}_{0} b \cos \beta_{0} .
$$

Substitution of (5.20) and (5.21) in (5.18) gives an expression for $\omega^{2}$

$$
\omega^{2}=\dot{\alpha}_{0}^{2}+\frac{1}{5}\left(14 \dot{\alpha}_{0} \dot{\gamma}_{0} \sin \beta_{0}+12 \dot{\gamma}_{0}^{2}-4 \tilde{g} \cos \beta_{0}\right),
$$

which is the frequency of small oscillations (5.16) around circular rolling motion. The rolling motion of a vertical disk on a straight line $\left(\beta_{0}=0\right.$ and $\left.\dot{\alpha}_{0}=0\right)$ is a special case. For this kind of rolling motion, the frequency is $\omega^{2}=-\frac{4}{5}\left(\tilde{g}-3 \dot{\gamma}_{0}^{2}\right)$, which is positive for a large enough rotation speed $\dot{\gamma}_{0}$. If $\dot{\gamma}_{0}$ is not large enough, then the gyroscopic terms are not able to make equilibrium with the gravitational terms, and the disk will fall. Similarly, for stationary rolling motion we obtain (using (5.13) and (5.15))

$$
\omega^{2}=\frac{4}{5} \tilde{g} \frac{4 \rho^{2}-1}{\rho \sqrt{\rho^{2}-1}}
$$

which is positive because $\rho>1$ for stationary rolling motion. 


\subsection{Feasibility of Circular Rolling Motion According to Coulomb's Law}

In Section 5.2, we considered circular rolling motion of the disk, which obeys the sticking condition (5.1). Considering such a motion, we are interested to predict, according to Coulomb's law of dry friction, whether the disk can remain in a sticking state. First, we derive from the Newton-Euler equations, the intensity $\left\|\boldsymbol{\lambda}_{T}\right\|$ of the tangential force of contact which acts in a circular rolling motion of the disk. The horizontal accelerations of the center of mass $S$ can be found as

$$
\begin{aligned}
& x_{S}=-\left(R-r \sin \beta_{0}\right) \sin \alpha_{0} \Rightarrow \ddot{x}_{S}=\dot{\alpha}_{0}^{2}\left(R-r \sin \beta_{0}\right) \sin \alpha_{0}, \\
& y_{S}=\left(R-r \sin \beta_{0}\right) \cos \alpha_{0} \Rightarrow \ddot{y}_{S}=-\dot{\alpha}_{0}^{2}\left(R-r \sin \beta_{0}\right) \cos \alpha_{0},
\end{aligned}
$$

where $x_{S}$ and $y_{S}$ are the first two components of ${ }_{I} \boldsymbol{r}_{O S}(t)$ according to Figure $8,{ }_{I} \boldsymbol{r}_{O S}(t)=$ $\left[\begin{array}{lll}x_{S}(t) & y_{S}(t) & z_{S}(t)\end{array}\right]^{\mathrm{T}}$. It follows from the Newton-Euler equations that

$$
m \ddot{x}_{S}=\lambda_{T x}, \quad m \ddot{y}_{S}=\lambda_{T y} \quad \Rightarrow \quad\left\|\boldsymbol{\lambda}_{T}\right\|=m \sqrt{\ddot{x}_{S}^{2}+\ddot{y}_{S}^{2}} .
$$

The equations (5.23) and (5.24) together with (5.25) yield to

$$
\left\|\boldsymbol{\lambda}_{T}\right\|=m\left(R-r \sin \beta_{0}\right) \dot{\alpha}_{0}^{2}=m\left(R-r \sin \beta_{0}\right) \rho^{2} \dot{\gamma}_{0}^{2},
$$

in which the pure rolling condition (5.11) has been used. The right-hand side of (5.26) can be recognized as the centrifugal force. Subsequently, by substituting in (5.26) the expression of $\dot{\gamma}_{0}^{2}$ given by (5.12), we obtain the intensity of the tangential contact force, which acts in a circular rolling motion,

$$
\left\|\boldsymbol{\lambda}_{T}\right\|=m R\left(1-\rho \sin \beta_{0}\right) \frac{4 \rho \tilde{g} \sin \beta_{0}}{\left(6-5 \rho \sin \beta_{0}\right) \cos \beta_{0}} .
$$

Because in circular rolling motion the height $z_{S}$ of the center of mass is constant, it follows from Newton's law that the normal contact force opposes the gravitation, i.e., $\lambda_{N}=m g$. According to Coulomb's law, for a friction coefficient $\mu_{T}>0$, a circular rolling motion is feasible if $\left\|\boldsymbol{\lambda}_{T}\right\| \leq \mu_{T} \lambda_{N}$ holds, i.e., if the indicator $\chi-$

$$
\chi=\left(1-\rho \sin \beta_{0}\right) \frac{4 \sin \beta_{0}}{\left(6-5 \rho \sin \beta_{0}\right) \cos \beta_{0}} \frac{1}{\mu_{T}}
$$

—fulfills the condition

$$
\chi \leq 1 .
$$

\subsection{Energy Decay during the Final Stage of Motion}

In the beginning of this analytical analysis, we studied the circular rolling motion (without dissipation) of a disk. As a special case, we focused on the stationary rolling motion, for which the center of mass $S$ remains immobile with respect to the inertial frame.

Section 4 shows the numerical simulation of a rolling disk for different types of friction models. The numerical results, in terms of $\boldsymbol{q}(t)$ (2.3) and $\boldsymbol{u}(t)$ (2.7), can be expressed in 
the parametrization ( $x, y, \alpha, \beta, \gamma)$ of the analytical model (see Figure 8). A number of observations can be made concerning the simulations with Coulomb and contour friction, Coulomb-Contensou friction and Coulomb-Contensou and contour friction (Sections 4.2 to 4.4). During the first phase of the motion, the disk rolls over the table much like the circular rolling motion. However, the system is not conservative due to the presence of friction, and the movement slowly changes. A short phase of rapid sliding occurs in the simulation with Coulomb and contour friction when $\chi$ has grown up to a value of about 1 (see (5.28)). The simulations with Coulomb-Contensou friction and Coulomb-Contensou and contour friction also show a peak of rapid sliding. We observe that the subsequent time-evolution of the disk is, for these three different mechanisms of dissipation, much like stationary rolling motion. As $\beta$ tends to $\pi / 2$, the center of mass hardly moves and the relative sliding velocity becomes small. Moreover, the component $\omega_{x}=\dot{\beta}$ of the angular velocity along the axis $\boldsymbol{e}_{x}^{R}$ (see Figure 8 ) becomes small when compared to the component $\omega_{y}=\dot{\gamma} \cos \beta$ along the axis $\boldsymbol{e}_{y}^{R}$.

In the following, we will study analytically the energy decay of a rolling disk for various kinds of dissipation using the following standing assumptions for the type of motion:

A.1 The center of mass is assumed to be almost immobile, i.e., $\rho \sin \beta=1$.

A.2 We assume $|\ddot{\beta}| \ll \tilde{g}$ and $|\dot{\beta}| \ll|\dot{\alpha}| \cos \beta$.

A.3 The sliding velocity is assumed to be small, i.e., $\dot{\alpha}=-\rho \dot{\gamma}$.

A. 4 We assume $\beta$ to be close to $\pi / 2$.

The analytically obtained energy decays for different kinds of dissipation will be compared with the energy decay during the final stage of the motion of the corresponding numerical simulations. With assumptions A.1-A.4, it follows from equation of motion (5.6) that (5.14) still holds approximately, i.e., $\dot{\gamma}(t)=\dot{\gamma}(\rho(t))$ and $\dot{\alpha}(t)=\dot{\alpha}(\rho(t))$ with $\rho(t)=1 / \sin \beta(t)$. Subsequently, we derive the total energy of the system $E=T+V$ with

$$
\begin{gathered}
T=\frac{1}{2} m_{K} \boldsymbol{v}_{S}{ }_{K}^{\mathrm{T}} \boldsymbol{v}_{S}+\frac{1}{2}{ }_{K} \boldsymbol{\omega}_{I B}{ }_{K}^{\mathrm{T}} \boldsymbol{\Theta}_{S K} \boldsymbol{\omega}_{I B} \\
=\frac{1}{2} m r^{2}\left((\dot{\alpha} \sin \beta+\dot{\gamma})^{2}+\dot{\beta}^{2}\right)+\frac{1}{2}\left(A \dot{\beta}^{2}+C(\dot{\alpha} \sin \beta+\dot{\gamma})^{2}+A \dot{\alpha}^{2} \cos ^{2} \beta\right), \\
V=m g r \cos \beta .
\end{gathered}
$$

Using the above assumptions, we approximate the total energy by the expression

$$
E=\frac{1}{2} A \dot{\alpha}^{2} \cos ^{2} \beta+m g r \cos \beta,
$$

in which only the major terms have been taken into account. An expression for the energy as a function of $\beta$

$$
E=\frac{3}{2} m r^{2} \tilde{g} \cos \beta
$$

follows from the substitution of (5.14) and $A=\frac{1}{4} m r^{2}$ in (5.32). In the following, we express, for different kinds of friction models, the power as a function of energy, i.e., $\dot{E}=f(E)$. The corresponding power-energy relations define a time-evolution of the system, which can be shown to verify the standing assumptions A.1-A.4. 
5.5.1. Contour Friction Model. First we consider a model of rolling friction called contour friction (see Section 2.3.3), which relates the velocity of the contact point on the contour of the disk $\gamma_{R}$ to a friction force by relation (2.33). Considering the parametrization of the disk $(x, y, \alpha, \beta, \gamma)$ introduced in Section 5.1, it holds that $\gamma_{R}=-r \dot{\gamma}$. If we choose a dry contour friction law, as used in Section 2.3.3, then the dissipation rate reads as

$$
\dot{E}=-\mu_{R} \lambda_{N}\left|\gamma_{R}\right|=-r \mu_{R} \lambda_{N}|\dot{\gamma}|
$$

The assumptions A.2 and A.4 allow us to approximate the normal contact force with $\lambda_{N}=m g$. We now have to express $\dot{\gamma}$ as a function of $E$. Using (5.14), (5.33), and $\rho=1 / \sqrt{1-\cos ^{2} \beta}$, it holds that

$$
\dot{\gamma}^{2}=\frac{4 \tilde{g}}{\rho \sqrt{\rho^{2}-1}}=\frac{4 \tilde{g}\left(1-\cos ^{2} \beta\right)}{\cos \beta}=\frac{4 \tilde{g}\left(1-\left(\frac{2 E}{3 m r^{2} \tilde{g}}\right)^{2}\right)}{\left(\frac{2 E}{3 m r^{2} \tilde{g}}\right)} .
$$

It follows from assumption A.4 that $E \ll \frac{3}{2} m r^{2} \tilde{g}$, and we approximate (5.35) with

$$
\dot{\gamma}^{2} \approx \frac{6 \tilde{g}^{2} m r^{2}}{E}=\dot{\alpha}^{2}
$$

The dissipation rate $\dot{E}$ for dry contour friction can therefore be expressed as a function of $E$,

$$
\dot{E}=-\frac{a}{\sqrt{E}}, \quad E>0,
$$

with $a=\sqrt{6} \mu_{R} m^{3 / 2} \tilde{g}^{2} r^{2}>0$ and constant. For an arbitrary initial condition $E\left(t_{0}\right)=E_{0}$, the differential equation (5.37) obeys the solution

$$
E(t)=\left(E_{0}^{\frac{3}{2}}-\frac{3}{2} a\left(t-t_{0}\right)\right)^{\frac{2}{3}} \quad \text { for } t_{0} \leq t \leq t_{f},
$$

which shows (see the black line in Figure 9a) a decrease to zero in a finite time $t_{f}-t_{0}=$ $2 E_{0}^{\frac{3}{2}} /(3 a)$.

If we consider a viscous contour friction model $\lambda_{R}=-c \gamma_{R}$, in which $c$ is the viscosity parameter, then the dissipation rate reads as

$$
\dot{E}=-c \gamma_{R}^{2}=-c r^{2} \dot{\gamma}^{2}
$$

Using the approximation (5.36), similar to the above analysis, we deduce that

$$
\dot{E}=-\frac{a}{E}, \quad \text { with } \quad a=6 c \tilde{g} m r^{3} .
$$

For an arbitrary initial condition $E\left(t_{0}\right)=E_{0}$, the differential equation (5.40) obeys the solution

$$
E(t)=\left(E_{0}^{2}-2 a\left(t-t_{0}\right)\right)^{\frac{1}{2}} \quad \text { for } t_{0} \leq t \leq t_{f},
$$

which shows (see the grey line in Figure 9a) a decrease to zero in a finite time $t_{f}-t_{0}=$ $E_{0}^{2} /(2 a)$. 


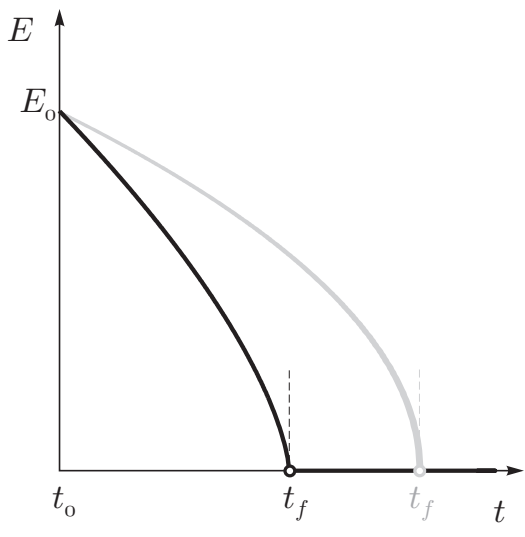

(e)

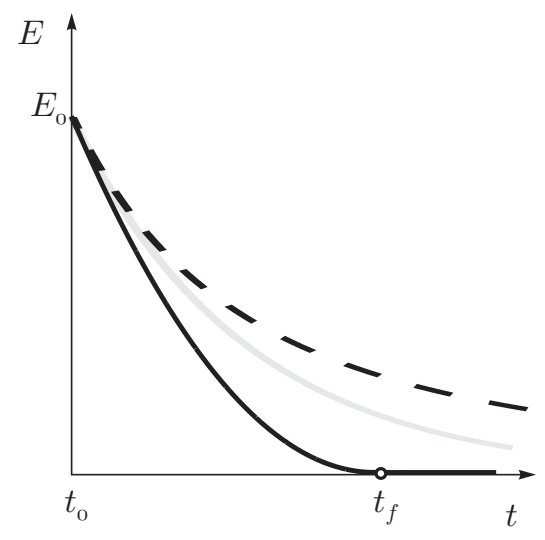

(f)

Fig. 9. Energy decay for (a) contour friction (dry = black, viscous = grey), (b) classical rolling friction $($ dry $=$ black, viscous $=$ grey $)$ and Contensou friction $($ dashed $)$.

5.5.2. Classical Rolling Friction Model. Classically, the resistance against rolling of two interacting bodies is modelled by (see [23]) a set-valued force law which relates the orthogonal projection, on the contact plane, of the relative angular velocity of the bodies $\boldsymbol{\omega}_{R}$, to a tangential frictional couple $\boldsymbol{M}_{R}$, transmitted by the contact

$$
-\boldsymbol{\omega}_{R} \in N_{C_{R}}\left(\boldsymbol{M}_{R}\right) \quad \text { with } \quad C_{R}:=\left\{\boldsymbol{v} \in \mathbb{R}^{2} \mid\|\boldsymbol{v}\| \leq \mu_{R} \lambda_{N}\right\} .
$$

Similar to the analysis conducted with the contour friction model, we study here the total energy decrease for classical rolling friction. More generally, the set $C_{R}$ will be some noncircular closed convex set corresponding to an anisotropic law. The projection of the angular velocity vector on the contact plane $\boldsymbol{\omega}_{R}$ can be decomposed along the axis $\boldsymbol{e}_{x}^{R}$ and $\boldsymbol{e}_{y}^{R}$ (see Figure 8)

$$
\omega_{R}=\left[\begin{array}{c}
\omega_{x} \\
\omega_{y}
\end{array}\right]=\left[\begin{array}{c}
\dot{\beta} \\
\dot{\gamma} \cos \beta
\end{array}\right] \text {. }
$$

The dissipation rate due to classical rolling friction reads as

$$
\dot{E}=-\mu_{R} \lambda_{N}\left\|\omega_{R}\right\|
$$

The assumptions A.1-A.4 allowed us to make the approximation

$$
\dot{E}=-\mu_{R} \lambda_{N}|\dot{\gamma}| \cos \beta \text {. }
$$

Substitution of (5.33) and (5.36) gives $\dot{E}$ as a function of $E$

$$
\dot{E}=-a \sqrt{E}, \quad \text { with } \quad a=\frac{2 \sqrt{6}}{3} \mu_{R} \tilde{g} \sqrt{m}=\text { const. }>0 .
$$

For an arbitrary initial condition $E\left(t_{0}\right)=E_{0}$, the differential equation (5.46) obeys the solution

$$
E(t)=\left(\sqrt{E_{0}}-\frac{a}{2}\left(t-t_{0}\right)\right)^{2} \quad \text { for } t_{0} \leq t \leq t_{f}
$$


which shows (see the black line in Figure $9 \mathrm{~b}$ ) a decrease to zero in a finite time $t_{f}-t_{0}=$ $2 \sqrt{E_{0}} / a$.

If we consider a viscous classical rolling friction model $\boldsymbol{M}_{R}=-c \boldsymbol{\omega}_{R}$, then the dissipation rate reads as

$$
\dot{E}=-c\left\|\omega_{R}\right\|^{2}=-c \dot{\gamma}^{2} \cos ^{2} \beta,
$$

in which assumption A. 2 has been used and $c$ is a viscosity parameter. Considering the approximation (5.36), similar to the above analysis, we deduce that

$$
\dot{E}=-a E, \quad \text { with } a=\frac{8 c}{3 m r^{2}} .
$$

For an arbitrary initial condition $E\left(t_{0}\right)=E_{0}$, the differential equation (5.49) obeys the solution

$$
E(t)=E_{0} e^{-a\left(t-t_{0}\right)} \quad \text { for } t \geq t_{0},
$$

which shows (see the grey line in Figure 9b) that an asymptotic behaviour of the energy occurs. Consequently, a decrease to zero is therefore not achieved in a finite time.

5.5.3. Coulomb-Contensou Friction. We assume the sliding velocity $\boldsymbol{\gamma}_{T}$ to be negligible. Consequently, the dissipation rate is due to work done by the drilling torque, i.e.,

$$
\dot{E}=\lambda_{\tau} \cdot \gamma_{\tau},
$$

which according to (2.22) and the Coulomb-Contensou friction law (Section 2.3.2) leads to

$$
\dot{E}=-\frac{3 \pi}{16} \mu_{F} m g \cdot \epsilon|\dot{\alpha}+\dot{\gamma} \sin \beta|,
$$

in which $\epsilon$ is the radius of the contact surface. Using (5.33), (5.36), $\rho \sin \beta=1$, and $\dot{\alpha}=-\rho \dot{\gamma}$, we deduce that

$$
\dot{E}=-a E^{\frac{3}{2}}, \quad \text { with } \quad a=\frac{\pi \sqrt{2}}{4 \sqrt{3}} \frac{\mu_{F} \epsilon}{r^{2} \sqrt{m}} .
$$

For an arbitrary initial condition $E\left(t_{0}\right)=E_{0}$, the differential equation (5.53) obeys the solution

$$
E(t)=\left(\frac{1}{\sqrt{E_{0}}}+\frac{a}{2}\left(t-t_{0}\right)\right)^{-2} \quad \text { for } t \geq t_{0},
$$

which shows (see the dashed line in Figure $9 b$ ) an asymptotic behaviour of the energy.

\section{Discussion of Analytical, Numerical, and Experimental Results}

In this section we will discuss the analytical and numerical results (Sections 4 and 5) and compare those with experimental results which can be found in the literature. 
The results of the simulation with Coulomb friction (Section 4.1) show that the pure rolling motion of the disk is accompanied by small oscillations around the global motion of the disk. An analysis of (possible) small oscillations in the vicinity of circular rolling motion of the system is presented in Section 5.3. The consideration of a particular harmonic solution (with frequency $\omega$ ) of the analytical model of a rolling disk leads to equation (5.22), which relates $\omega^{2}$ to the position and velocity parameters of the system. The application of this relation, using the positions and velocities from the numerical results, gives an almost constant frequency $\omega=5.7 \mathrm{~Hz}$. This value is in good accordance with the frequency of the nutation, which can be observed in the graph $(t, \beta)$ of Figure 4 . Therefore, these oscillations have a physical meaning and are not numerical artifacts. Although the study presented in Section 5.3 is based on the consideration of a conservative system, the relation (5.22) appears to give at each time-instant a good approximation for the frequency of the small nutational oscillation $(t, \beta)$ observed in the numerical results for the (nonconservative) Coulomb and contour friction model (Section 4.2), CoulombContensou friction model (Section 4.3), and Coulomb-Contensou and contour friction model (Section 4.4).

The analysis on the analytical model of Section 5.4 reveals that according to Coulomb's law, for a friction coefficient $\mu_{T}>0$, a circular rolling motion is feasible if the indicator $\chi$ (5.28) fulfills the condition $\chi \leq 1$. The indicator $\chi$ has been evaluated for each timestep of the numerical simulation with the Coulomb friction model (Section 4.1). The parameter $\rho$, involved in the definition of $\chi$, has been evaluated for each timestep according to (5.11) by taking the computed values of $\dot{\alpha}$ and $\dot{\gamma}$. It appears that the indicator $\chi$ remains smaller than 1 , which agrees with the fact that no sliding occurs during this simulation (see Figure 4). The time-evolution of $\chi$ for the simulation with Coulomb and contour friction (Section 4.2) is shown in Figure 10. A good accordance between $\chi(t)$ and $\boldsymbol{\gamma}_{T}(t)$ can be observed. The sticking phases correspond to values of $\chi<1$, and the sliding phases correspond to values of $\chi>1$. As mentioned in Section 4.2, the last part of the motion, $32 \mathrm{~s}<t<67 \mathrm{~s}$, computed with Coulomb and contour friction consists of short alternating stick and slip phases. For this part of the motion, the indicator $\chi$ oscillates around the value $\chi=1$ (depicted by the dashed line in Figure 10). The motion is much like stationary rolling motion, for which the center of mass practically remains immobile (notice the point $(-2.63,-1.89)$ in the $\left(x_{S}, y_{S}\right)$ graph of Figure 5) and for which the disk slowly flattens due to dissipation. Of course, we have to bear in mind that $\chi$ has been defined for the analytical model with circular rolling motion, under the assumptions that $\dot{\beta}=0, \dot{x}=0$, and pure rolling of the disk (no slip of the contact point). The numerical simulations with Coulomb friction and with Coulomb and contour friction are characterized by $\dot{\beta}(t) \ll 1, x(t) \approx$ const. and $\left\|\boldsymbol{\gamma}_{T}(t)\right\| \ll 1$ for almost all $t$. The assumptions for circular rolling motion are therefore approximately fulfilled, which explains the good accordance between the $\chi$ and $\boldsymbol{\gamma}_{T}$. Consequently, the parameter $\chi$ is a useful tool to indicate whether the disk will remain sticking.

In the analytical analysis of Section 5.5, we studied the energy decrease of a rolling disk under the assumptions A.1-A.4 for various types of friction. If dry contour friction is assumed, then the analytical analysis indicates that the energy decreases in a finite time to zero, which is in accordance with the numerical simulation for Coulomb and contour friction (see the $(t, E)$ graph of Figure 5). Moreover, we checked that the profile of $E(t)$ 


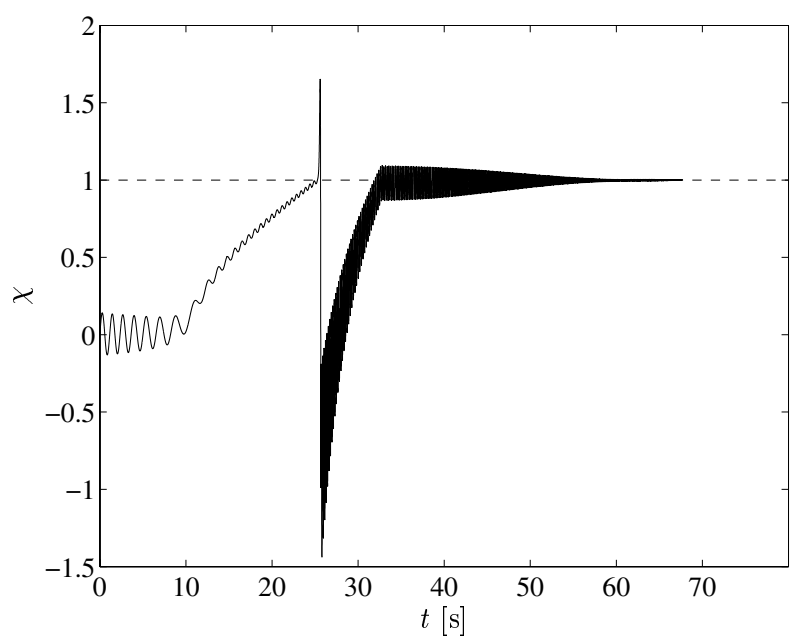

Fig. 10. Time-evolution of the indicator $\chi$ for simulation with Coulomb and dry contour friction.

during the final stage of the corresponding simulation is indeed of the form (5.38). If Coulomb-Contensou is assumed, then the analytical analysis indicates that the energy decreases asymptotically to zero, which is in accordance with the numerical simulation for Coulomb-Contensou friction (see Figure 6) and the profile of $E(t)$ is indeed of the form (5.54). Additional simulations, not presented here, show that the numerical results for classical rolling friction are in accordance with the analytical results of Section 5.5.2. Moreover, a classical rolling friction model was used by [11] for the simulation of a rolling disk, and their numerical results show an asymptotic energy profile which is in agreement with our analytical results. The final stage of the motion of the simulation with Coulomb-Contensou and contour friction is similar in form to the final stage of the simulation with contour friction. This can be understood from the analytical analysis of the energy decay (Section 5.5): The dissipation rate $\dot{E}$ (5.37) due to contour friction increases with decreasing energy $E$, while the dissipation rate $\dot{E}$ (5.53) due to CoulombContensou friction decreases with decreasing energy $E$. The final stage of the motion with both Coulomb-Contensou and contour friction will therefore be dominated by the dissipation due to contour friction.

Experiments on rolling disks have been performed by [15] and [7], both presenting their results in terms of $\dot{\alpha}(t)$. McDonald and McDonald [15] performed the experiment on a single disk using a phototransistor. Easwar et al. [7] measured the movement of steel disks and a steel ring on supports of various materials using a high-speed camera. The experimental data of [15] and [7] can be described for the final stage of the motion by a power law,

$$
\dot{\alpha}(t) \propto\left(t_{f}-t\right)^{-\frac{1}{n_{\text {exp }}}} .
$$

The experiments of [15] suggest that $n_{\exp }=4$, and the experiments of [7] on various disk/ring-support combinations suggest that $2.7<n_{\exp }<3.2$. 
The analytical analysis of Section 5.5 leads to relation (5.36) between $\dot{\alpha}$ and $E$,

$$
\dot{\alpha}(t) \propto E(t)^{-\frac{1}{2}} \propto\left(t_{f}-t\right)^{-\frac{1}{n_{\text {ana }}}} .
$$

We now derive $n_{\text {ana }}$ for those friction models of Section 5.5, which lead to an energy decease to zero in a finite time. It follows from (5.38) that $n_{\text {ana }}=3$ for the dry contour friction model. Similarly, it holds that $n_{\text {ana }}=4$ for viscous contour friction. Dry classical rolling friction leads to $n_{\text {ana }}=1$. If we now compare the experimental results with the results from the analytical model, then dry or viscous contour friction could well explain the energy decay of the experimental results. We therefore believe that contour friction is indeed the dominant mechanism of dissipation during the final stage of the motion. However, we have to keep in mind that (at least in theory) other dissipation mechanisms might exist that lead to a similar energy decay.

\section{Conclusion}

In this paper a numerical model for a rolling disk has been developed, using a parametrization with Euler parameters, which is able to take into account the unilateral contact constraints and different types of frictional models. The numerical model has proven its capability to describe the motion of objects, with a flat side of circular contour, on a plane. The numerical results seem to be reasonable for the chosen contact parameters, but a fairly small stepsize has to be taken to properly describe the motion.

An analytical analysis of the energy decay during the final stage of rolling motion has been performed in Section 5. The derived energy profiles for the different types of frictional dissipation mechanisms agree well with the corresponding numerical energy profiles. An energy decrease to zero in finite time occurs for dry contour friction and dry classical rolling friction and, remarkably, for viscous contour friction. An asymptotic decrease to zero of the energy occurs for viscous classical rolling friction and (dry) Contensou friction. The analytical analysis of Section 5 gives a better understanding of the behaviour of the disk during the last stage of the numerical simulations. A comparison with available experimental results suggests that contour friction might very well be the dominant mechanism of dissipation.

Goodwine and Stépán [9] study the control of the classical shimmying wheel by feedback linearization techniques. Coulomb (sliding) friction was used by [9] to explain the shimmying phenomenon. It would be of interest to study the classical shimmying wheel under the influence of spatial Coulomb-Contensou friction, i.e., a combined sliding and pivoting friction model. The interaction between pivoting and sliding might change the stability of the nonholonomic system.

The results obtained in this paper may provide a good basis for numerical treatment of more general dynamical multicontact problems involving interaction between cylindrical and planar objects and the like.

\section{References}

[1] Alart, P., and A. Curnier. A mixed formulation for frictional contact problems prone to Newton-like solution methods. Computer Methods in Applied Mechanics and Engineering 92 (1991), 353-375. 
[2] Alart, P., and M. Renouf. Un nouvel algorithme de quasi-optimisation pour la résolution des problèmes multicontacts et application aux milieux granulaires. Actes du $16^{\text {ème }}$ Congrès Français de Mécanique (2003).

[3] Appell, P. Sur l'intégration des équations du mouvement d'un corps pesant de révolution roulant par une arête circulaire sur un plan horizontal; cas particulier du cerceau. Rendiconti del Circolo Matematico di Palermo 14 (1900), 1-6.

[4] Borisov, A. V., I. S. Mamaev, and A. A. Kilin. Dynamics of rolling disk. Regular and Chaotic Dynamics 8, 2 (2003), 201-212.

[5] Brogliato, B. Nonsmooth Mechanics, 2d ed. Springer, London, 1999.

[6] Chaplygin, S. A. On the motion of a heavy body of revolution on a horizontal plane (in Russian). Physics Section of the Imperial Society of Friends of Physics, Anthropology and Ethnographics 9, 1 (1897), 10-16.

[7] Easwar, K., F. Rouyer, and N. Menon. Speeding to a stop: The finite-time singularity of a spinning disk. Physical Review E 66, 045102(R) (2002).

[8] Glocker, Ch. Set-Valued Force Laws, Dynamics of Non-Smooth Systems, vol. 1 of Lecture Notes in Applied Mechanics. Springer-Verlag, Berlin, 2001.

[9] Goodwine, B., and G. Stépán. Controlling unstable rolling phenomena. Journal of Vibration and Control 6 (2000), 137-158.

[10] Jean, M. The nonsmooth contact dynamics method. Computer Methods in Applied Mechanics and Engineering 177 (1999), 235-257.

[11] Kessler, P., and O. M. O'Reilly. The ringing of Euler's disk. Regular and Chaotic Dynamics 7, 1 (2002), 49-60.

[12] Korteweg, D. J. Extrait d'une lettre à M. Appell. Rendiconti del Circolo Matematico di Palermo 14 (1900), 7-8.

[13] Leine, R. I., and Ch. Glocker. A set-valued force law for spatial Coulomb-Contensou friction. European Journal of Mechanics-A/Solids 22 (2003), 193-216.

[14] Leine, R. I., and H. Nijmeijer. Dynamics and Bifurcations of Non-Smooth Mechanical Systems, vol. 18 of Lecture Notes in Applied and Computational Mechanics. Springer-Verlag, Berlin-Heidelberg-New York, 2004.

[15] McDonald, A. J., and K. T. McDonald. The rolling motion of a disk on a horizontal plane. Preprint, Princeton High School, New Jersey (2001).

[16] Moffatt, H. K. Euler's disk and its finite-time singularity. Nature 404 (2000), 833-834.

[17] Moreau, J. J. Bounded variation in time. In Topics in Nonsmooth Mechanics, J. J. Moreau, P. D. Panagiotopoulos, and G. Strang, eds. Birkhäuser Verlag, Basel, Boston, Berlin, 1988, pp. 1-74.

[18] Moreau, J. J. Unilateral contact and dry friction in finite freedom dynamics. In Nonsmooth Mechanics and Applications, J. J. Moreau and P. D. Panagiotopoulos, eds., vol. 302 of CISM Courses and Lectures. Springer, Wien, 1988, pp. 1-82.

[19] Moreau, J. J. Some numerical methods in multibody dynamics: Application to granular materials. European Journal of Mechanics-A/Solids 13, 4 (1994), 93-114.

[20] Moreau, J. J. Modélisation et simulation de matériaux granulaires. In Actes du $35^{\text {ème }}$ Congrès National d'Analyse Numérique (June 2003). CD-ROM, to appear.

[21] O'Reilly, O. M. The dynamics of rolling disks and sliding disks. Nonlinear Dynamics 10, 3 (1996), 287-305.

[22] Parviz, E. N. Computer-aided analysis of mechanical systems. Prentice-Hall International Editions, London, 1988.

[23] Pérès, J. Mécanique générale. Masson \& $\mathrm{C}_{\mathrm{ie}}$, Paris, 1953.

[24] Stanislavsky, A. A., and K. Weron. Nonlinear oscillations in the rolling motion of Euler's disk. Physica D 156 (2001), 247-259. 